\title{
Considering distribution grids and local flexibilities in the prospective development of the European power system by 2050
}

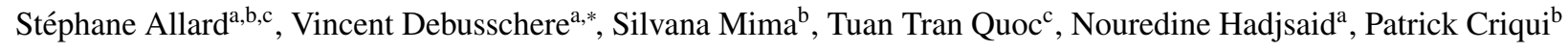 \\ ${ }^{a}$ Univ. Grenoble Alpes, CNRS, Grenoble INP ${ }^{1}$, G2Elab, 38000 Grenoble, France \\ ${ }^{b}$ GAEL, CNRS, Grenoble INP ${ }^{1}$, INRA, Univ. Grenoble Alpes, 38000, Grenoble, France \\ ${ }^{c}$ CEA-INES, Univ. Grenoble Alpes, 73370 Le Bourget-du-Lac, France
}

\begin{abstract}
Previous works proposed a tool coupling models of a prospective outlook on long-term energy systems and a transmission grid investment and dispatch, focusing on the representation of the European transmission grid and its development on the horizon 2050-2100. In this paper, this prospective tool is further improved with the capacity to compute voltage as well as active and reactive power flows at the level of the distribution grid. This added capacity allows analyzing various issues related to the integration of variable energy resources in three representative real medium voltage distribution grids (urban, rural and semi-urban). Technical flexibility solutions such as on-load tap changers, variable energy resources curtailment and storage technologies are modeled and compared to reinforcement. A cost comparison between these flexibility solutions is also carried out. Finally, the new version of the tool is used to evaluate the $\mathrm{CO}_{2 \text {-eq }}$ emissions linked to the development of the European power system infrastructure, with flexibility solutions, up to the year 2050 (both high voltage alternative and direct current lines reinforcement being considered) under a $2{ }^{\circ} \mathrm{C}$ climate energy policy scenario. Results show that it exists various options for the development of the European grid infrastructure, which are clearly sensitive to the level of accuracy in the representation of the physical infrastructures and their technical limitations. Being able to represent the distribution grid, in addition to the transmission one, has a noticeable impact on the prospective outlook of the European power systems both in terms of infrastructure reinforcement and estimation of the needs of flexibility solutions.
\end{abstract}

Keywords: Long-term planning, European transmission grid, distribution grids, variable energy resources, storage systems, flexibility solutions

\section{Introduction}

Distribution grids were not designed for the integration of variable renewable energy sources (vREs) [1]. As a consequence, a number of new problems appear currently, that the distribution system operators (Dso) must tackle [2].

\footnotetext{
${ }^{*}$ Corresponding author

Email address: vincent.debusschere@grenoble-inp.fr (Vincent Debusschere)

${ }^{1}$ Institute of Engineering Univ. Grenoble Alpes
} 
Among other, there are violations of voltage limits [3], and reverse power flows [4]. Combined with power quality problems, new protection schemes must be designed to avoid disturbances in the electricity supply [5]. The amount of energy can also surpass the grid capacities and the Dso face situations of congestion especially in rural distribution grids.

Even if not all VRES is connected at the distribution level, most issues related to the vREs integration are observed at the level of the distribution grids, which are then key to model in the long-term energy models. In prospective scenarios, the associated costs to this integration could be underestimated.

\section{Integrating distribution grids in long-term prospective models at the European scale}

\subsection{Context}

There is a strong need to develop adequate tools to assess the impacts of vREs integration in transmission and more particularly in distribution grids and evaluate the relevance of various existing or new solutions [6]. Usually, simulation models are first set up to understand the influence of the vREs integration on the quality of supply, for example voltage harmonics [7]. However, the studies are generally case-specific: a given distribution grid is usually described, and the solutions are analyzed to solve the identified issues on a short-term basis. Based on the different observations, new control systems are proposed to avoid the various issues [8]. Other articles focus on the use of flexibility options to help the integration of vREs through the minimization of a cost function. Battery energy systems strategies are modeled and applied in real distribution grids [9]; similarly, demand response and electric vehicles charging are used to avoid congestion [10]. Reconfiguration of the distribution grids seems also to be a strategy for higher penetration of VRES [11]. One of the main limitations of these studies is the robustness of the results. The use of stochastic models can be a solution as deterministic models can underestimate the investments needs [12]. Consequently, a lot of research is being conducted to study the integration of VRES into the distribution grid in short-term perspectives.

As pointed out in [13], on a larger scale, for instance pan-European or even larger, very few long-term energy models actually implement electricity dispatch and decision investments in the transmission grid [14]. Also, in many models, the grid is considered as a "copper plate" (i.e. no constraints are taken into account for power flows) [15]. Hence, there is a linear relation between investments in vREs capacities and investments in upgrading or extending the transmission grid: for each MW of vREs installed, they assume an increase of transmission grid investments [16]. In this context, it is worth mentioning two long-term models: TIMEs [17] and PRIMEs [18], which both include a power sector and a transmission grid. However, the transmission grid evolution is exogenous, set by the user, and they do not have a physical representation of the distribution grids. They rather have a cost approach: the demand and production are allocated to each voltage level. The flows are calculated based on transmission and distribution grids costs [19].

Previous works have been conducted on the Prospective Outlook on Long-term Energy Systems (POLEs) model [20], which has been coupled with a dedicated dispatch module called European Transmission Grid Investment and 
Dispatch (EUTGRID) [21]. In this coupling, EUTGRID aims at optimizing the power system operations and includes investments in the transmission grid while PoLEs decides, among other things, new investments in power plant capacities at the (supra)-national level. The EUTGRID model focuses on the representation of the European transmission grid and its development. A major hypothesis is that there is no congestion within a region. But this is only true if the sub-transmission and the distribution grids are correctly sized for any kind of (drastic) event.

To the best of our knowledge, none of the described models currently implement a representation of a distribution grid. The main issues (faced as well in this paper) are contradictory. First, in order to run a pan-European model with a full representation of distribution grids, complete data is usually not available. Second, the computational burden will increase significantly once the representation of the distribution grids is added. The representation of the transmission grid is still simplified in the current state of art of long-term perspective models, so one can imagine that integrating a full representation of distribution grids has not yet been considered.

\subsection{Distribution grids and modeling hypothesis}

Multiple data-sets describing distribution grids are openly available to analyze issues within the power system (usually on short-term scenarios). Focusing on the representation of the European distribution grids, a project called "LV grid Solutions" with the University of Manchester made available 25 detailed models of low-voltage grids from Manchester [22]. These data-sets could be used with EUTGRID, but the representation of the medium voltage is lacking, and it is only limited to urban areas.

The Distribution System Operators Observatory from the European Joint Research Center collected data on European distribution systems and released 13 representative distribution grids with various voltage levels [23]. In this data-set, three grids are geo-referenced and have the following characteristics: urban, semi-urban and rural. Figure 1 shows the resulting geo-referenced representative grids to better apprehend their relative size and distribution. The main difference is related to the distance between each node: the rural grid has long lines (up to $10 \mathrm{~km}$ ) while the urban one has shorter lines (not exceeding $1 \mathrm{~km}$ ). The semi-urban grid represents an intermediate situation where a major portion of the grid has short lines with some long lines. These grids have a connection with the high-voltage grid (through a transformer). The three geo-referenced grids are used in this paper to represent urban, semi-urban and rural distribution grids. Their characteristics allow us to compare them based on vREs integration, grid infrastructure, voltage limitations notably.

\subsection{Problem statement}

EUTGRID [25] was further improved and the new developments are presented in this paper. It consists in representing voltage and power flows (active and reactive) at the distribution grids levels. This new model is then used to analyze the various issues related to the integration of VREs in three representative distribution grids (urban, semiurban and rural). Technical solutions such as on-load tap changers (OLTC), reinforcement, vres curtailment and storage technologies are considered. A cost comparison between these solutions is also carried out, from the grid perspective, 


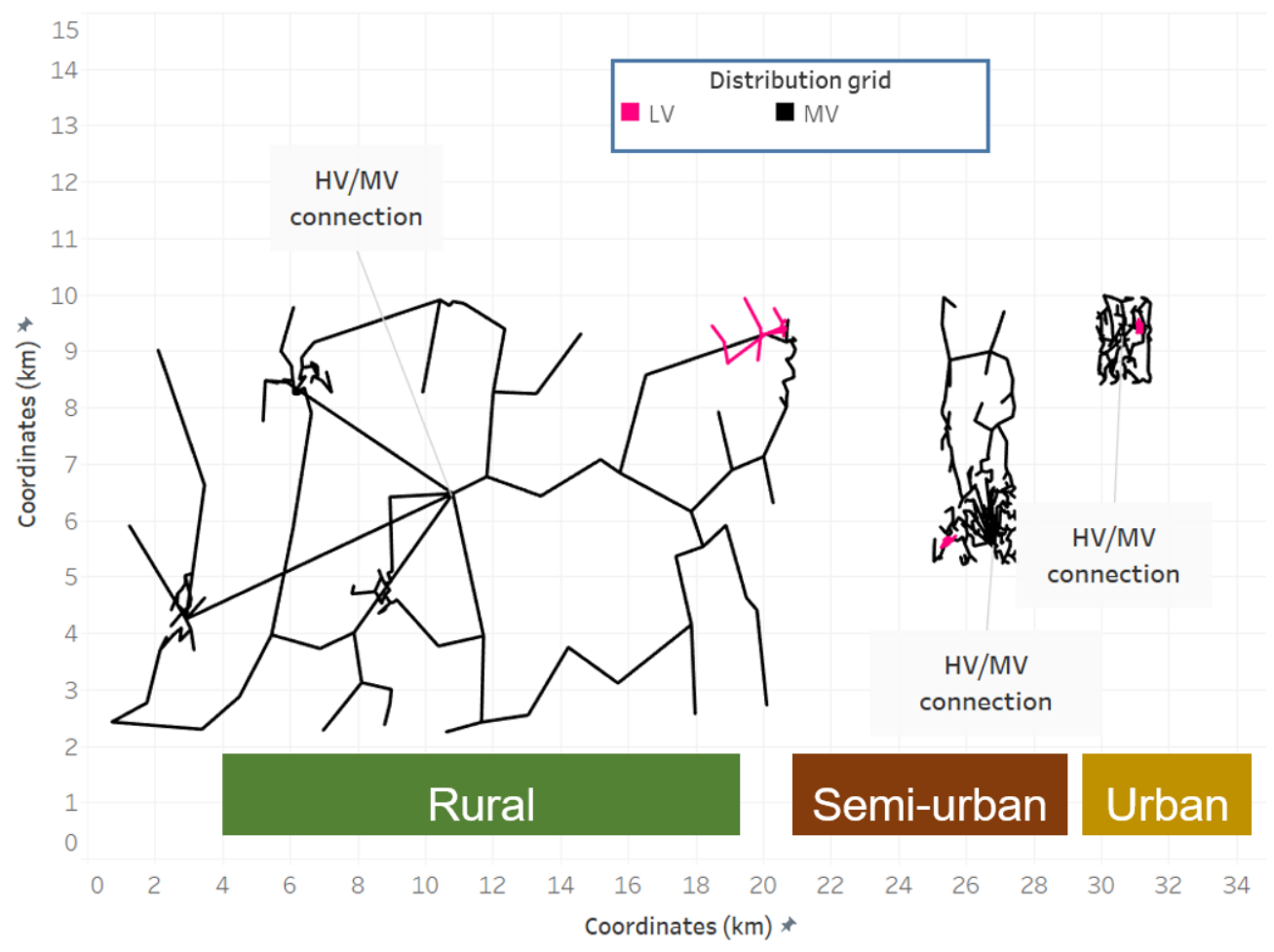

Figure 1: Geographical representation of the distribution grids implemented in EUTGRID, based on [24]. Left: rural; center: semi-urban and right: urban. 
not the retailers [26]. Finally, the new version of EUTGRID, called European Transmission Grid and Dispatch with Distribution grid representation (EUTGRID\&D), is coupled with POLES and the impacts on the equivalent $\mathrm{CO}_{2 \text {-eq }}$ emissions, the flexibility options and the evolution of the transmission grid are discussed. The interaction with the transmission grid is based on computations run on three representative nodes located in France, rather than the entire European power system, due to computational limitations (duration of the simulations).

\section{Calculation methods}

\subsection{POLES and EUTGRID}

EUTGRID incorporates a unit commitment and dispatch tool, implemented as an optimal power flow (OPF), that minimizes the total operating costs of the system on a 24 hours basis under various constraints: the supplied power at each node must be equal to the demand, transmission grid power flows must respect the maximum thermal line capacities, ramping capabilities, minimum and maximum generation capacities by technology, etc. For each simulated year, representative load days (for summer and winter) and representative vREs production days are used per cluster of nodes (i.e. countries and regions of countries). In EUTGRID\&D, the voltage and the reactive power are also considered for the distribution grids. EutGRID does not have any market model as this is implemented in POLEs, which decides the investments in the power plants based on the expected evolution of demand and their use in EUTGRID.

The coupling is presented in Figure 2 and described as follows [21]: at year $N$, poles provides the state of the power system with installed capacities, electricity demand at the level of the states. EutGRID computes the operation of the power sector with that information. Then, the output data are send back to POLES which can make the investments decisions for the next year, $N+1$. EuTGRID integrates a transmission capacity module to decide new investments in the transmission grid. However, with the fast and large-scale integration of vREs capacities, a double phenomenon must be taken into account: location of future bottlenecks need to be anticipated while at the same time, actual congestions must be resolved as installed capacities differ from expectations. The expected capacities are determined by poLEs with myopic simulations.

To capture these two types of planning, the mechanism consists of a 3 year rolling window. At the beginning of this 3 year period, transmission grid investments are calculated based on expected installed capacities for the next 10 years: we name it "anticipation planning". Then at the end of the 3 year period, the investments needs are determined using actual installed power plant capacities in order to solve congestions.

\subsection{Linearized power flow}

The EUTGRID model contains in fact an oPF simplified as a DC load flow for the transmission system. But once we integrate distribution grids in this long-term prospective model, even if we consider the scale of the European power system, reactive powers cannot be neglected and a (more complex) Ac load flow must be implemented. Thus, for each time step, the complete power flow equations for active power $P_{k}$ and reactive power $Q_{k}$, for each node $k$ (and $j$ ), 


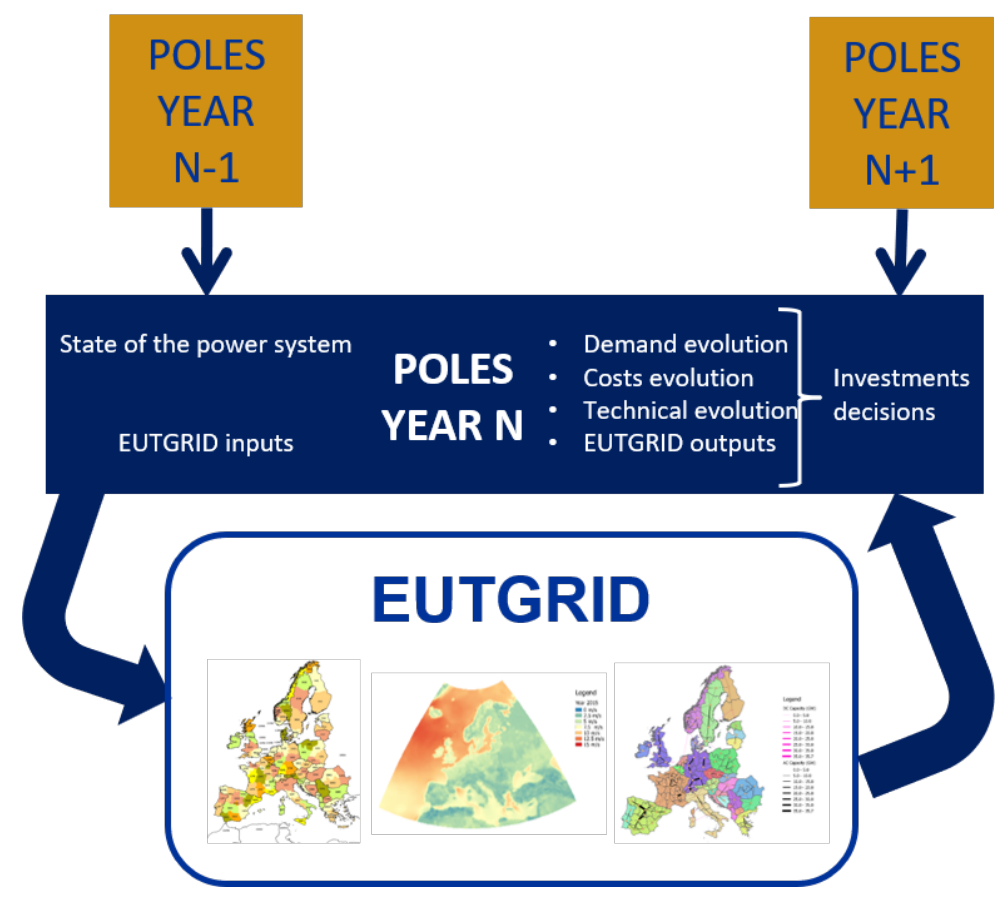

Figure 2: Recursive representation of the set of modeling tools used as a basis for the work, coupling the Prospective Outlook on Long-term Energy Systems (pole) and the European Transmission Grid Investment and Dispatch (EUTGRID) [21].

depending of the bus admittance $Y$ (its real part $G$ and its imaginary part $B$ ), the angles $\theta$ and voltage $V$ are expressed in (1).

$$
\forall(k, j)\left\{\begin{array}{l}
P_{k}=\sum_{j}\left|V_{k} \| V_{j}\right|\left(G_{k \leftrightarrow j} \cos \left(\theta_{k}-\theta_{j}\right)+B_{k \leftrightarrow j} \sin \left(\theta_{k}-\theta_{j}\right)\right) \\
Q_{k}=\sum_{j}\left|V_{k} \| V_{j}\right|\left(G_{k \leftrightarrow j} \sin \left(\theta_{k}-\theta_{j}\right)-B_{k \leftrightarrow j} \cos \left(\theta_{k}-\theta_{j}\right)\right)
\end{array}\right.
$$

In (2), the active power at each node $k$ is related to the sum of powers flowing from the neighboring nodes $i$.

$$
\forall(k, i) ; P_{k}=\sum_{i \neq k} P_{k \leftarrow i}=\sum_{i \neq k}\left|V_{k} \| V_{i}\right|\left(G_{k \leftrightarrow i} \cos \left(\theta_{k}-\theta_{i}\right)+B_{k \leftrightarrow i} \sin \left(\theta_{k}-\theta_{i}\right)-\left|V_{k}\right|^{2} \times G_{k \leftrightarrow i}\right)
$$

In the distribution grid, the difference between angles is considered small thus (2) can be approximated by (3).

$$
P_{k \leftarrow i} \approx\left|V_{k}\right|\left(G_{k \leftrightarrow i}\left(\left|V_{k}\right|-\left|V_{i}\right|\right)+\left|V_{i}\right| B_{k \leftrightarrow i}\left(\theta_{k}-\theta_{i}\right)\right)
$$

The final assumption, used in [27], considers that the voltage magnitude at each node does not deviate too much from the nominal value $V_{\text {nom }}$ but that the difference between two voltages has more effect on power flows. Therefore, (3) can be replaced with (4), using a per-unit system, with $V_{\text {nom }}$ taken as the base voltage (1 p.u.).

$$
P_{k \leftarrow i} \approx V_{\text {nom }}\left(G_{k \leftrightarrow i}\left(\left|V_{i}\right|-\left|V_{k}\right|\right)+V_{\text {nom }} B_{k \leftrightarrow i}\left(\theta_{k}-\theta_{i}\right)\right)
$$


Similarly, reactive power flow in the line $k \leftrightarrow i$ can be approximated using the same hypotheses, as described in (5).

$$
Q_{k \leftarrow i} \approx V_{n o m}\left(B_{k \leftrightarrow i}\left(\left|V_{k}\right|-\left|V_{i}\right|\right)+V_{n o m} G_{k \leftrightarrow i}\left(\theta_{k}-\theta_{i}\right)\right)
$$

\subsection{Constraints on the apparent power}

The active and reactive power flows are linked with the non-linear equations where $S_{k \leftarrow i}$ is the apparent power of the considered line $k \leftrightarrow i$. This apparent power is limited by the transmission capacity of the line, $S_{k \leftarrow i}^{\max }$.

$$
S_{k \leftarrow i}=P_{k \leftarrow i}^{2}+Q_{k \leftarrow i}^{2} \leq S_{k \leftarrow i}^{\max }
$$

The linearization of (6) follows the method described in [27]. The flows can be represented in a $P / Q$ diagram, reported in Figure 3. The apparent power flow is limited by the dotted circle. Two sets of constraints are introduced to linearize the apparent flow limitations. First, based on (6), the active and reactive power flows are limited by the apparent power, as expressed in (7). It corresponds to the lines $P= \pm 1$ and $Q= \pm 1$ in Figure 3.

$$
\left\{\begin{array}{l}
-S_{k \leftarrow i}^{\max } \leq P_{k \leftarrow i} \leq S_{k \leftarrow i}^{\max } \\
-S_{k \leftarrow i}^{\max } \leq Q_{k \leftarrow i} \leq S_{k \leftarrow i}^{\max }
\end{array}\right.
$$

The final approximation corresponds to the restrictions of the areas in the corners of the squares, expressed in (8).

$$
c_{\alpha}^{P} \times P_{k \leftarrow i}+c_{\alpha}^{Q} \times Q_{k \leftarrow i} \leq \sqrt{2} S_{k \leftarrow i}^{\max }
$$

These four constraints are determined by the parameters $c_{\alpha}^{P}$ and $c_{\alpha}^{Q}$, which describe the four quadrants presented in Figure 3. However, these approximations have some limitations. Some areas which are not allowed, only considering the non-linear equations, can still be accessed after simplification.

The linear equations representing the Ac load flow are implemented in GAMs using the solver CPLEx. Consequently, EUTGRID with these new set of equations now forms a new model, called European Transmission Grid and Dispatch with Distribution grid representation (EUTGRID\&D). The presented power flow has been successfully compared with the package MATPOWER which provides steady-state power system simulation [28].

\section{4. $\mathrm{CO}_{2-e q}$ emissions calculation}

The calculation of $\mathrm{CO}_{2-\mathrm{eq}}$ emissions in each region uses the hypotheses of poLEs: only the fossil production is considered to emit $\mathrm{CO}_{2-\mathrm{eq}}$ emissions. Within the fossil production, production from coal pollutes more than production from oil. Finally, production from gas is the cleanest energy. The different carbon content for each fossil fuel is taken from POLES' database [20]. 


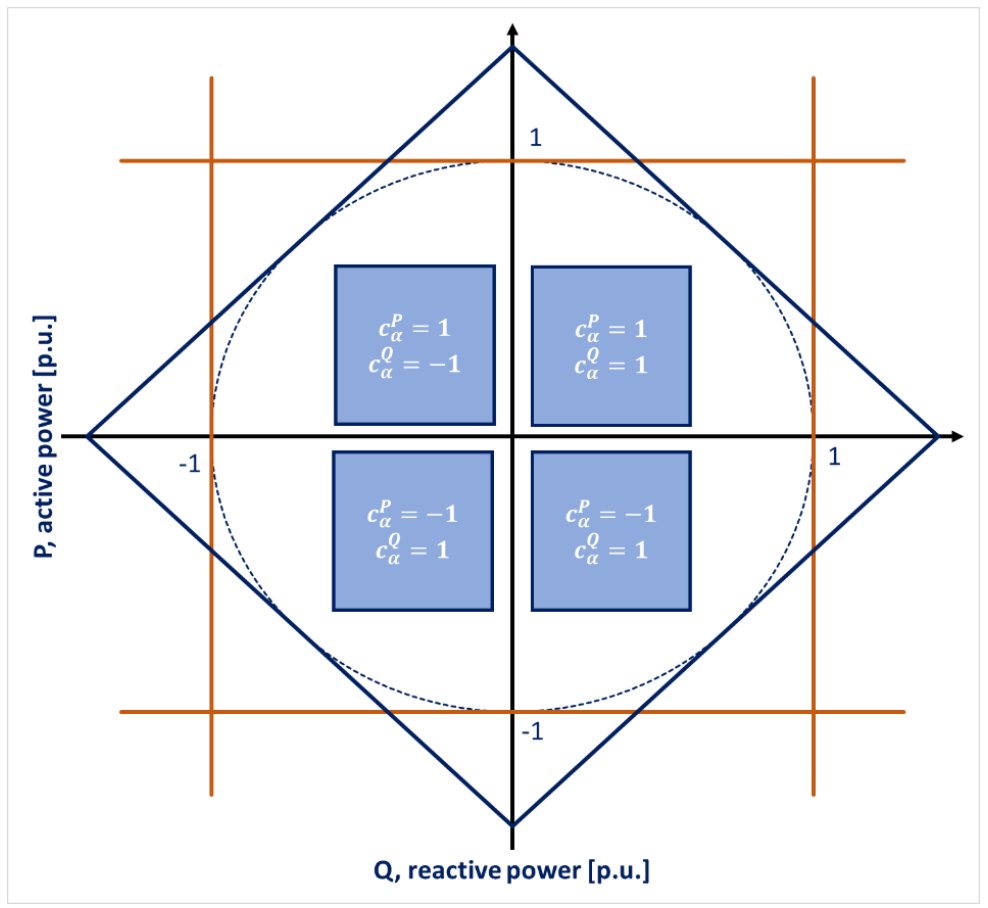

Figure 3: Graphical representation of the apparent, active and reactive power flow limitation in EUTGRID\&D, based on [27].

The emissions in a region are calculated for the energy supplied in this particular region without taking into account import and export [29]. The total local emissions $E_{i}^{\text {tot }}$ is calculated using the $\mathrm{CO}_{2 \text {-eq }}$ emissions factors and the local production for each fuel.

$$
\forall i ; E_{i}^{\text {tot }}=C_{\text {coal }} \times P_{i}^{\text {gas }}+C_{\text {oil }} \times P_{i}^{\text {oil }}+C_{\text {gas }} \times P_{i}^{\text {gas }}
$$

\section{Flexibility levers for distribution grids}

\subsection{Grid data}

A set of equations describing the linearized Ac load flow is included together with the representation of three real distribution grids to EUTGRID\&D (rural, urban and semi-urban). The rural distribution grid is connected to a French node located in the South of France. This region has been chosen because its local energy mix contains more solar PV production. The semi-urban distribution grid is connected to a French node located in the North of France. This region has been chosen because it concentrates more wind production than PV capacities. Finally, the urban distribution grid is connected to the densest French node located, in Ile-de-France (where you find the capital, Paris).

The only producing technologies available at the level of distribution grids are supposed to be onshore wind and decentralized Pv. Storage technologies are also included but they are limited to stationary batteries, vehicle-to-grid and grid-to-vehicle [30]. All those technologies are assumed to be distributed according to the density of the population 
except for wind capacities. Wind capacity is indeed installed at the medium voltage level, rather than at the low voltage level. Following that statement, wind turbines are installed in less populated areas where social acceptance is likely to be higher.

In the end, these distribution keys help drawing more characteristics per category of distribution grid. The allocation in percentage is represented for the urban, semi-urban and rural grid in Figure 4, including the allocation of wind capacities and the allocation of demand.

In urban areas (Figure 4b), the demand is equally distributed among all nodes. In the rural grid (Figure 4f), the loads are more dispersed, with six groups of nodes where most of the demand is concentrated, corresponding to small settlements. The semi-urban grid (Figure 4d) shares the characteristics of urban and rural grids with uniform demand distribution in the urban portion and small demand in the rural part.

Because of the complexity of these grids, they have been simplified so that the dispatch can be solved in EUTGRID\&D in a reasonable time. Low-voltage customers and vREs producers are aggregated to their nearest medium-voltage node. However, for each representative grid, one low-voltage grid has been included in the European-scale model to draw conclusions for that voltage level as well.

\subsection{VRES integration capacities}

An analysis is performed on the three grids to characterize the issues related to large-scale integration of vRES. Various solutions, such as OLTC, reinforcement, storage or vREs curtailment are modeled to solve the identified issues and help reaching a high share of vREs production. Finally, the costs of these solutions are calculated and compared in scenarios of VRES integration.

\subsubsection{Without flexibility levers}

The rising share of VRES modifies the operation of the three considered grids, mostly regarding over-voltages and non-distributed energy. The analysis of the various issues is carried out for the three representative distribution grids. The share of solar production is gradually increased in each distribution grid and for every percentage, EUTGRID\&D computes the dispatch and the resulting voltage values in the grid. The maximum voltages for the medium-voltage and low-voltage grids are thus retrieved. In the low-voltage grid, the maximum voltage values are always greater than the ones in the medium-voltage grid: there is a second transformer, which connects the medium-voltage to the low-voltage and increases the voltage, in per unit. The results are gathered in Figure 5.

In Figure 5, the maximum voltage increases faster in the low-voltage grid for all grids. It can be observed that the rural low-voltage is the most sensitive to the integration of solar PV with an important rise after a solar penetration rate of $25 \%$. It almost reaches the upper limit of 1.1 p.u. In the medium-voltage grids, only the rural distribution grid sees a visible increase in voltage from 1.047 p.u. up to 1.058 p.u. This violation of the limit of 1.05 p.u. occurs when around $30 \%$ of the demand is covered by solar Pv. For the urban and semi-urban distribution grids, congestions occur 

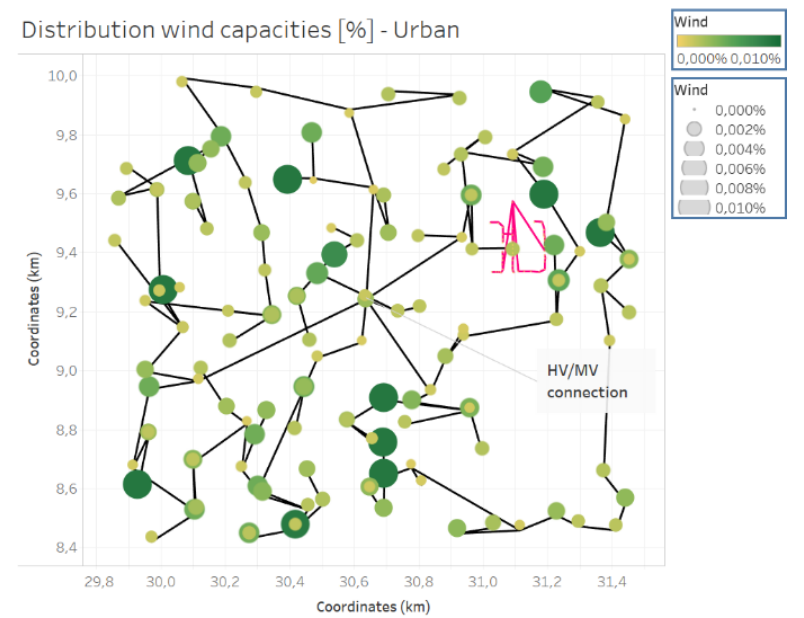

(a) Installed wind capacities in the urban distribution grid.
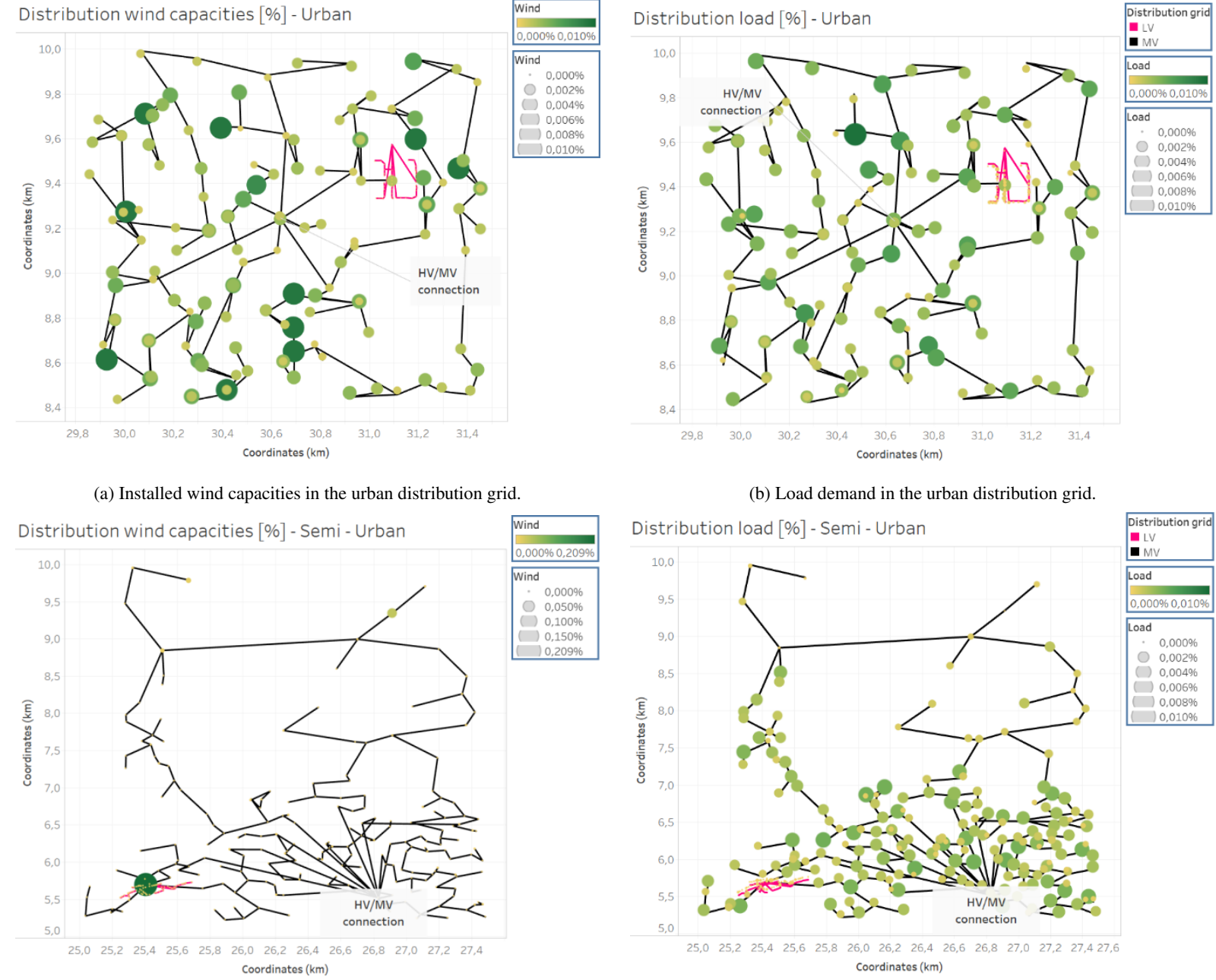

(b) Load demand in the urban distribution grid. Distribution load [\%] - Semi - Urban
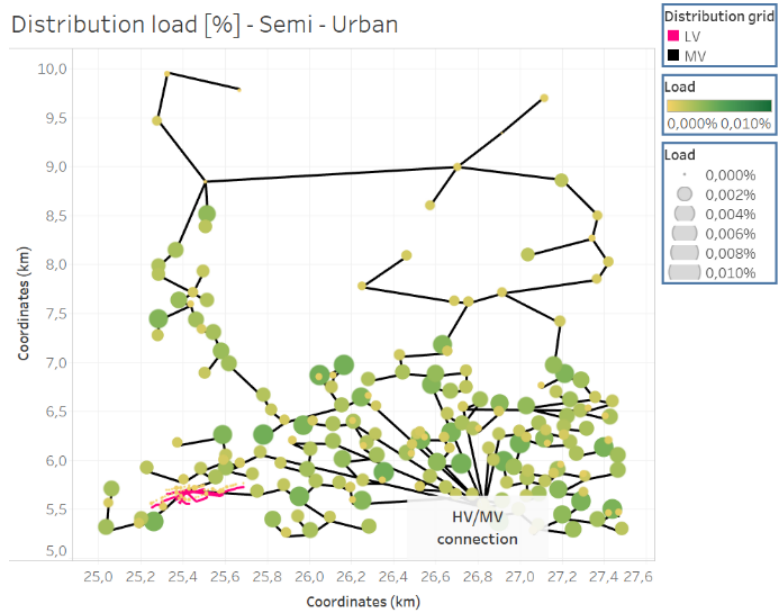

(c) Installed wind capacities in the semi-urban distribution grid.

Distribution wind capacities [\%] - Rural
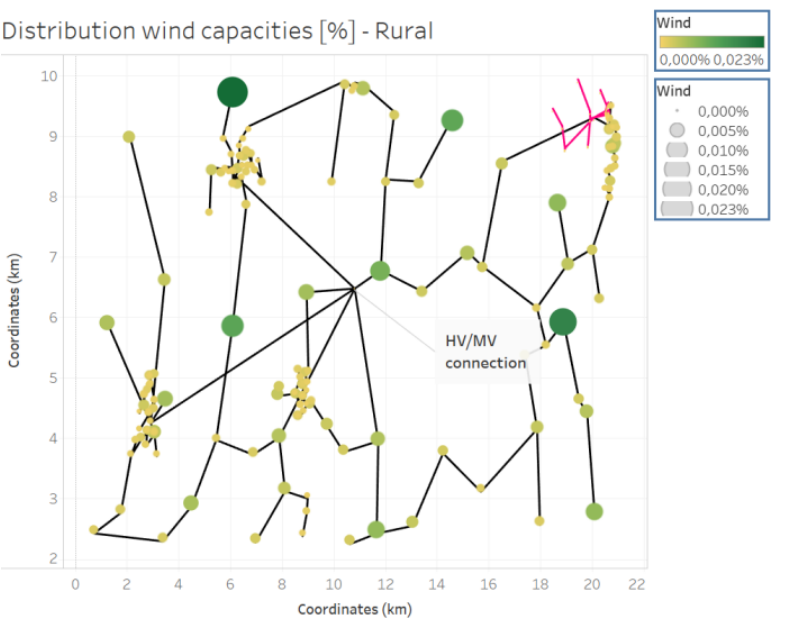

(e) Installed wind capacities in the rural distribution grid.

(d) Load demand in the semi-urban distribution grid.

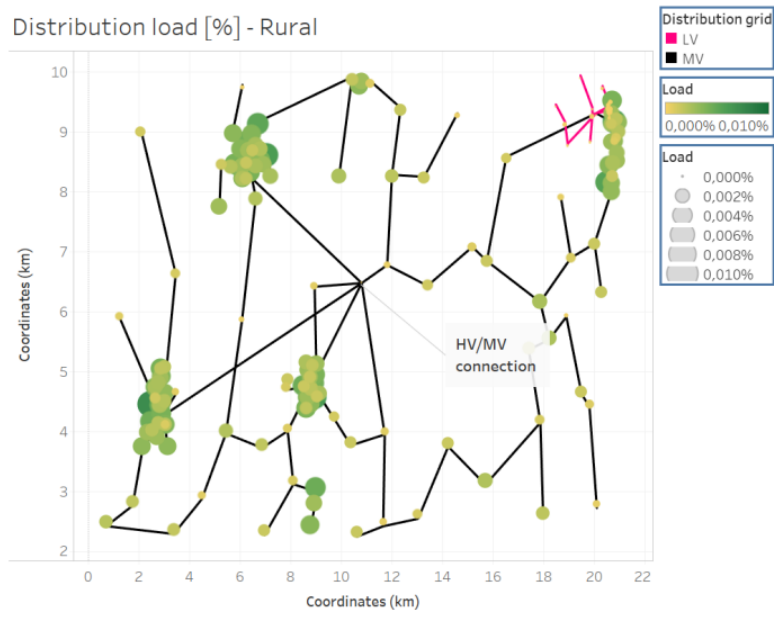

(f) Load demand in the rural distribution grid.

Figure 4: Graphical representation of the modeling hypothesis of local consumption and production in the three considered distribution grids. 


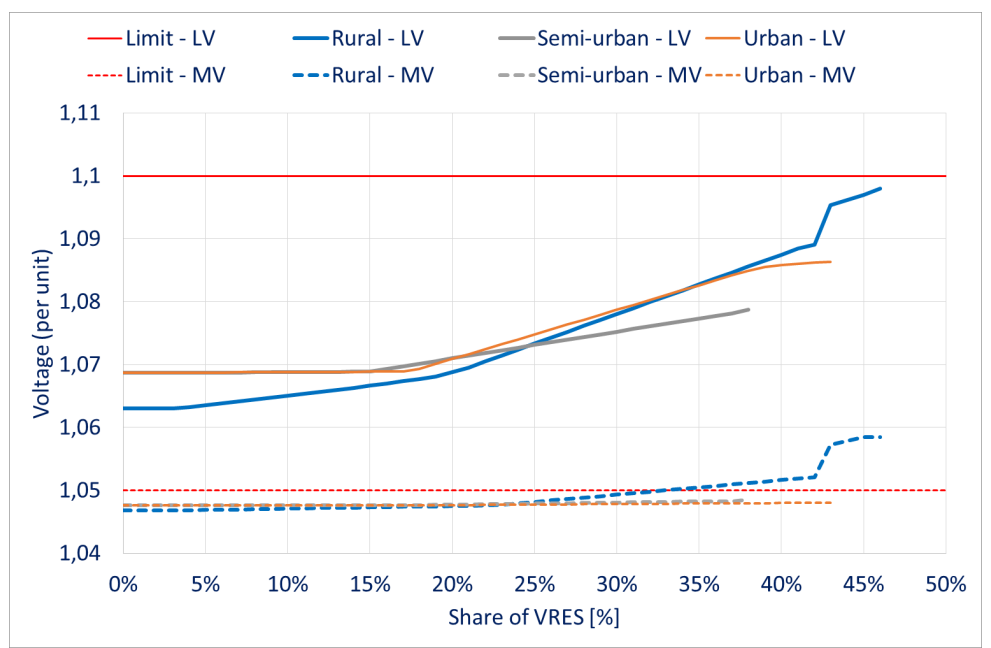

Figure 5: Maximum voltage for each type of distribution grid and each voltage level as a function of the local solar production rate.

before over-voltages when the share of $\mathrm{PV}$ production reaches almost $40 \%$. That is also the reason why the voltage values are not shown in Figure 5 above $45 \%$ of local Pv production.

In a situation with only local solar Pv, the rural distribution grid sees a greater increase of voltage level than in the other representative distribution grids, especially at the end of long lines. If the share of vREs keeps increasing above $45 \%$, congestions appear in the rural distribution grid. After $47 \%$ of the demand, non-distributed energy (NDE) appears at every node representing around $4 \mathrm{MWh}$, which is equivalent to $1.8 \%$ of the demand of the considered grid. In Figure 6, the amount of NDE at each node of the grid is represented in kWh together with the percentage of its yearly demand. It can be observed that almost all nodes have a similar level of NDE but in the low-voltage grid, it represents only $0.4 \%$ of the demand.

\subsubsection{With flexibility levers}

To facilitate the integration of vREs in the distribution grids, the voltage and the congestions issues must be tackled. Various solutions exist which act directly at the voltage level such as the oltc or reinforcement. Another group of solutions acts on the energy production to reduce voltage. It can also reduce congestions. It includes vREs curtailment and storage technologies.

Acting directly on the voltage. Voltage control can be achieved with the use of an olTc. The olTc modifies its tap ratio to maintain the voltage at the secondary winding of the transformer within the accepted limits.

In EUTGRID\&D, a lossless olTC with 9 taps is implemented. The tap ratio can change the voltage using the following percentages $[-7.5 ;-5 ;-3.5 ;-1.75 ; 0 ; 1.75 ; 3.5 ; 5 ; 7.5]$. The olTc, as a replacement of a simple transformer, is suitable to some solve under- and over-voltage situations, not all.

With an implemented oltc, the maximum voltages for low-voltage and medium-voltage grids are retrieved for each percentage of solar $\mathrm{PV}$ integration in the rural distribution grid in Figure 7 . Once the share of solar production 


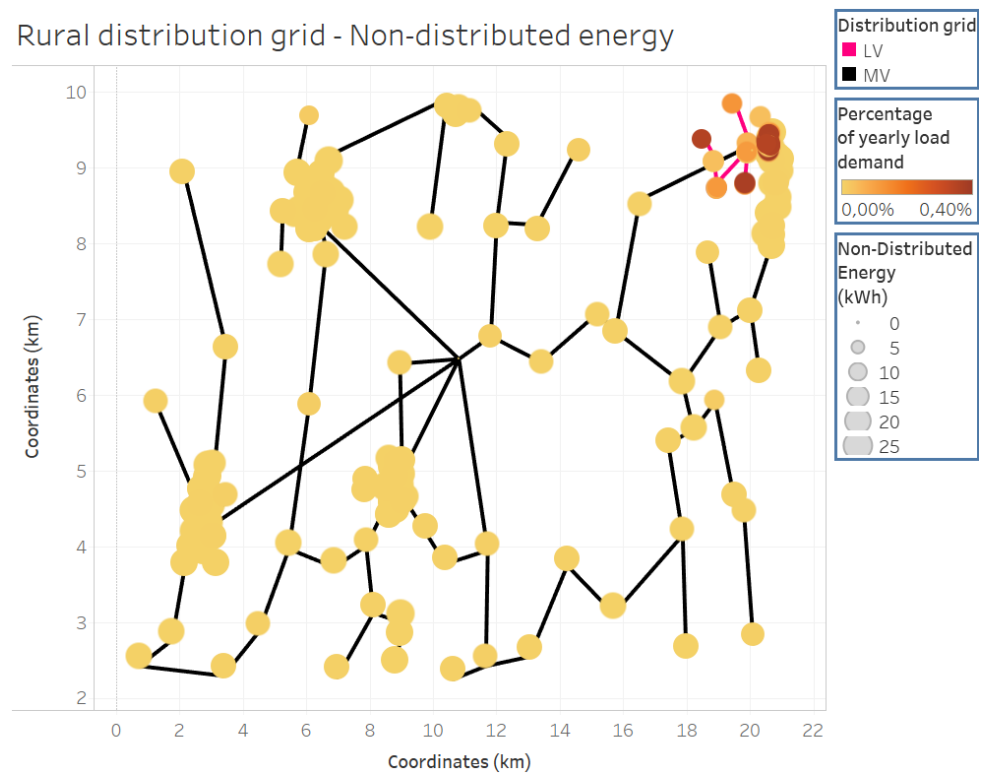

Figure 6: Non-distributed energy in the rural distribution grid (share of solar production: $47 \%$ of the demand).

exceeds $30 \%$, the olTc modifies its tap ratio and maintains the voltage at the medium-voltage grid under 1.05 p.u. until the share reaches $45 \%$. It can be observed that the voltage at the low-voltage grid stays around 1.08 p.u. and when the share of solar production reaches $44 \%$, the maximum voltage increases up to $1.086 \mathrm{p}$.u. The consequence is that the oLTC must modify more often its tap ratio as the PV penetration rate increases. Figure $7 \mathrm{~b}$ shows a bar graph with the number of yearly modifications versus the penetration rate of PV. When the share of vres is around $30 \%$, only 35 changes of tap ratio are required while with a share of $45 \%$, the number of changes is 10 times higher. However, congestions still appear when the share of solar production increases too much. Those cannot be solved with the only use of OLTCS.

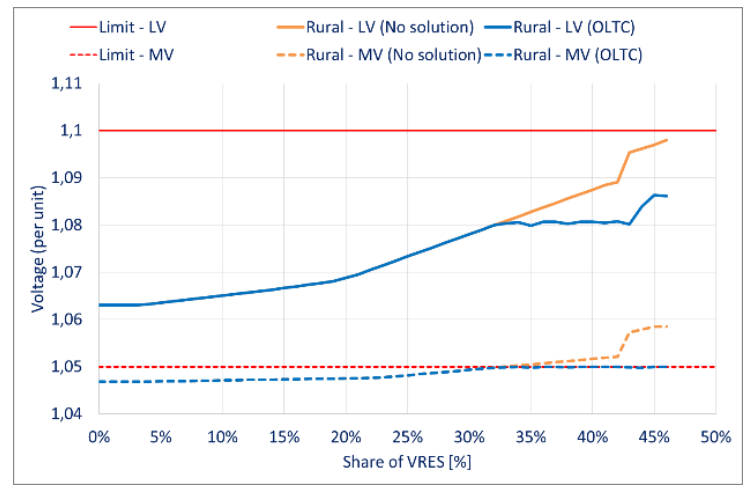

(a) Maximum voltage before and after operating oLTcs.

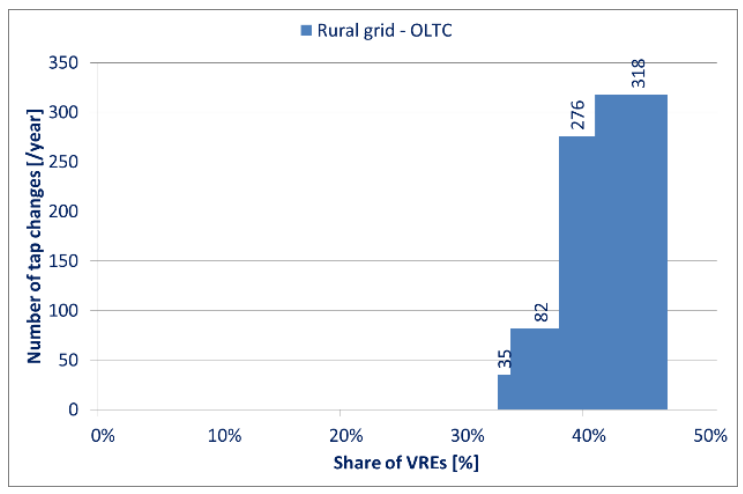

(b) Number of yearly modifications of tap ratio.

Figure 7: Impact of using otrcs in the rural grid as a function of the local solar Pv penetration rate.

Another way to reduce the over-voltage situation is to reinforce the grid. It has also the advantage of alleviating 
the congestions. The grid investment mechanism of EUTGRID [21] was use in EUTGRID\&D with some differences: it is only restricted to the distribution grids and the most congested lines are reinforced until there are no more NDE, without considering any payback period.

The resulting effect of the reinforcement is a significant reduction of the maximum voltage (below limits). However, the mean value of the voltage is slightly greater than the mean value of the initial state. It is a consequence of a better grid which allows more power flows and lower voltage drops from the transformer up to the end of the lines.

The method of reinforcement described here is a simplified version of reinforcement studies which require a detailed description of the grid and an adequate planning of these reinforcements [31]. Within the described framework, all the representative distribution grids manage to integrate the rising solar production up to $100 \%$ while keeping the voltage within limits. However, the reinforcements needs differ between each other. For each percentage of solar integration, EUTGRID\&D reinforces the grid and the results of these needs are illustrated in Figure 8.

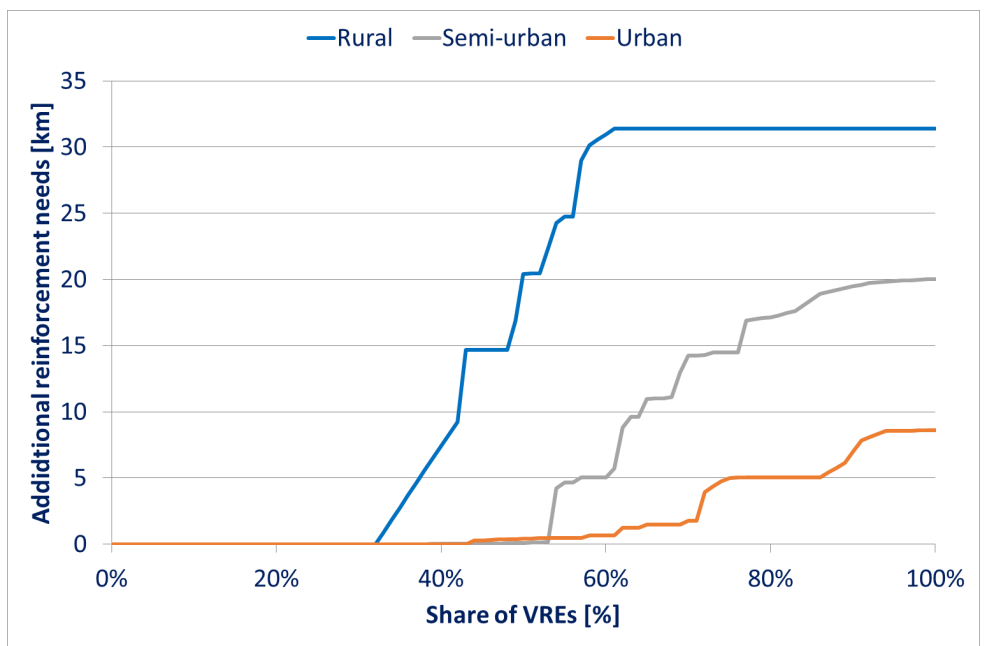

Figure 8: Reinforcement needs for the three distribution grids with only solar production.

For the three distribution grids, the length of the reinforcement rises as the integration of solar increases. Because of its long lines, the rural grid must reinforce more than $30 \mathrm{~km}$ and these needs increase steadily from $30 \%$ of share of VRES up to $60 \%$. After this point, no more additional reinforcements are needed. Concerning the urban grid, there are fewer reinforcements thanks to its robustness and its shorter lines. Before $70 \%$ of PV integration, almost no reinforcements are made, representing less than $2 \mathrm{~km}$. However, after that penetration rate, the grid reinforcement needs increase two more times, at $70 \%$ and $90 \%$ to reach around $10 \mathrm{~km}$ of additional lines. The semi-urban distribution grid combines the characteristics of the two previous ones, with additional requirements: starting to reinforce at $40 \%$ of $\mathrm{PV}$ penetration rate to reach up to $20 \mathrm{~km}$ of reinforcements in the end. These requirements are the consequences of its longer lines. 
Acting on energy production. As the solar production provokes over-voltages in the distribution grid, curtailing the production is another way to limit those violations. The main drawback of curtailing the vres production is that it is lost. In Figure 9, the percentage of energy curtailed is shown for the three distribution grids. The lost energy follows an exponential curve as the share of vRes increases. In the rural grid, $80 \%$ of the solar energy must be shed to cover $100 \%$ of the yearly demand. For the urban and semi-urban grids, around $20 \%$ must be shed. Therefore, in this rural grid, if VRES curtailment is the only solution considered, new VRES capacities will probably never be installed: either the curtailment is not reimbursed, and the losses will be too important for producers, or the curtailment is reimbursed then other cheaper solutions should be considered.

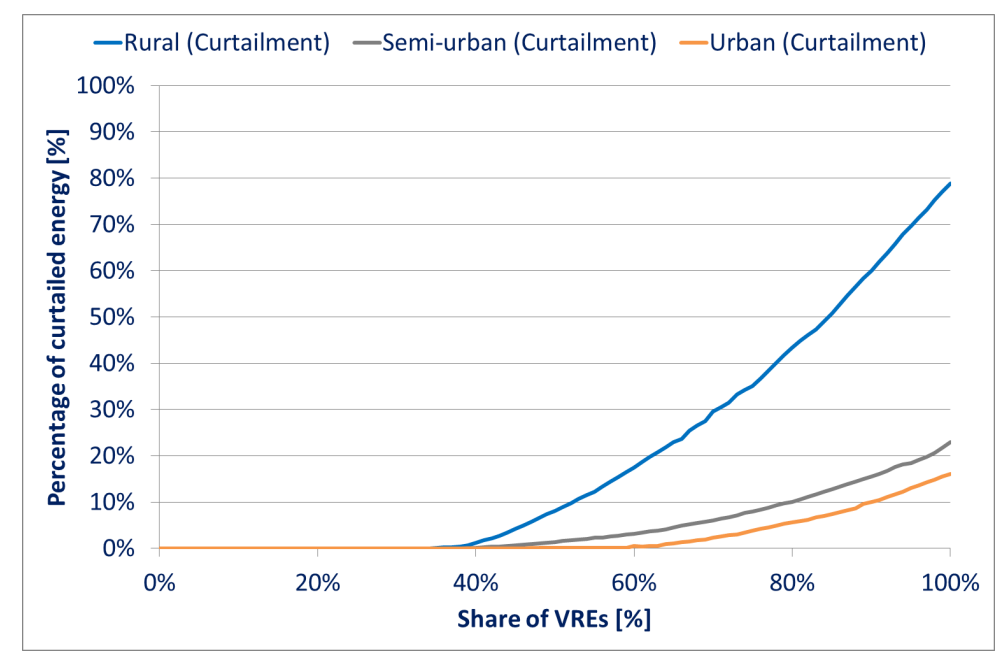

Figure 9: Yearly energy curtailment as a function of the PV penetration rate for the three distribution grids.

From the important decreases of the prices of PV modules, a new trend begins to emerge, which consists of oversizing the solar panels [32]. Increasing the size of the panels and the overall costs (including fixed and variable ones) enables the PV panels to produce during more hours while reaching its maximum power earlier in the day (in that case, it could reach injection limits at the grid level). Therefore, it produces more energy than a PV panel whose maximum peak does not exceed that limit. In this context, the increase in produced energy, induced by the over-sizing, exceeds the resulting curtailed energy and could justify its usage.

Storage systems. Another solution would be to use storage systems that charge the surplus of energy from vRES sources and discharge it when it is the most needed. In EUTGRID\&D, for simplification purposes, battery capacities correspond to lithium-ion technologies and are distributed following the population. Moreover, the installed capacities are equally increased until there is no more NDE in the distribution grid. During the period with high solar production, the batteries are being charged to alleviate the congestions and the over-voltage. Otherwise, because of the implemented strategies, storage capacities are being discharged during the low solar production and during off-peak hours. Note that batteries must have the same state of charge at the beginning and at the end of the day. 
Figure 10 shows the relation between the energy charged by storage technologies and the vREs energy produced for the distribution grids. The results show that the amount of energy charged by batteries increases in all grids. Compared to the curtailment of vREs, the charging of battery capacities represents less energy, with less than $20 \%$ of the vREs energy produced.

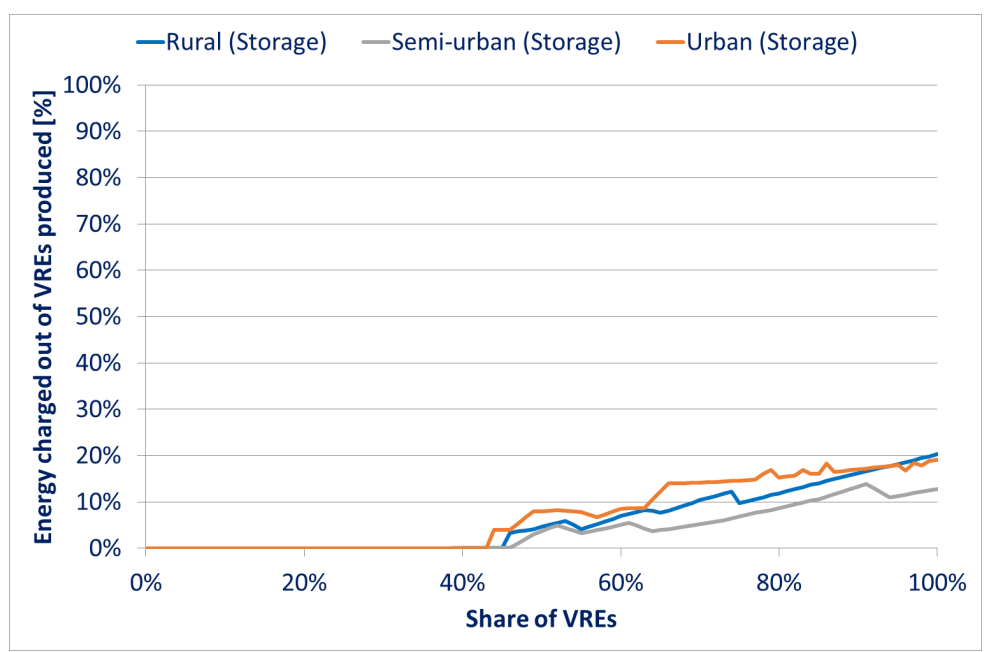

Figure 10: Percentage of energy stored compared to the yearly vREs production for the representative distribution grids (\%).

Cost parameters. To understand in which conditions the presented solutions can be preferred, a technical and economic analysis is performed. All parameters are listed in Table 1 and the associated hypothesis described below.

OLTC - It is only implemented in the rural grid as it is the only grid where over-voltages occur. It has also been assumed that it was already installed. Therefore, there are no initial investment costs. The maintenance cost corresponds to half of the total investment for the transformer.

Reinforcement - In distribution grids, the cost of reinforcement mostly depends on the length of the lines and not the capacity needs. The value of the investments also varies with the typology of the grids. The reinforcements do not need any maintenance during the 30-year scenario and a line is reinforced only once during the simulations. Therefore, the planning anticipates the needs.

Curtailment - The results have shown that the amount of curtailed energy could be very important, and it could slow down the integration of solar energy in the energy system. Therefore, the curtailment is assumed to be reimbursed by the distribution system operators and in this case-study, the French feed-in tariff is used.

Storage - The storage technologies consist of stationary lithium-ion batteries. The costs are decomposed in: investments costs for each installed $\mathrm{kW}$ and usage costs for charging. The technical lifetime of the installed batteries is set to 15 years [33]. 
Table 1: Costs parameters for the modeled solutions (source for the storage data: POLEs).

\begin{tabular}{llc}
\hline Solution & Parameter & Value \\
\hline \multirow{2}{*}{ OLTC } & Transformer rating & 80 MVA [34] \\
& Cost (new transformer) & $11.9 \mathrm{k} \$ / \mathrm{MVA}[34]$ \\
& Operations before maintenance & $10,000[34]$ \\
\hline \multirow{2}{*}{ Reinforcement } & Rural reinforcement & $96 \$ / \mathrm{m} \mathrm{[35]}$ \\
& Urban and semi-urban reinforcement & $150 \$ / \mathrm{m} \mathrm{[35]}$ \\
\hline \multirow{2}{*}{ Curtailment } & Cost (lost energy) & $200 \$ / \mathrm{MWh} \mathrm{[36]}$ \\
\hline \multirow{2}{*}{ Storage } & Capacity costs & $136 \$ / \mathrm{kW}$ \\
& Usage costs & $2.15 \$ / \mathrm{MWh}$ \\
\hline
\end{tabular}

Note that the actual cost benefit analysis based on data presented in Table 1 should include all available flexibility levers. However, in a first approach and to better illustrate the advantages and disadvantages of each considered modeling precision, extreme scenarios where investigated where only one flexibility is considered. In a second step, in Section 5.4, all the flexibility levers are considered when coupling POLES and EUTGRID\&D in a global optimization. With this latter approach, we are closer to reality, but it is more difficult to analyze the impact of each flexibility separately. In the end, POLES and EUTGRID\&D always make their decisions based on an economic optimization under technical constraints.

\subsubsection{Flexibility solutions for VRES integration in the distribution grids}

The associated net present value can be calculated for each solution, notably based on Table 1 . The discount rate is set to $8 \%$, as proposed by the French Regulatory Commission of Energy. The various paths of solar integration are assessed using a Monte-Carlo method. It was also assumed that the share of vres always increases to reach the targeted percentage. In this analysis, for each targeted percentage, around 500,000 unique scenarios were randomly selected: it corresponds to a margin of error of $0.23 \%$ with a confidence level of $99.9 \%$.

Monte Carlo assessment. Figure 11a shows the mean present value per solution and per distribution grid, corresponding to the share of VRES at the end of the 30-year period. Because the oLTC has never been replaced, there is no associated cost and it is not visible on this figure. For the three distribution grids, three sections can be identified, which are more noticeable in the rural distribution grid. The first section begins from $0 \%$ to $\sim 35 \%$ : no flexibility is needed. Then from $\sim 35 \%$ to $\sim 65 \%$, the flexibility solutions have similar costs. It can be noticed that the cost for the curtailment exponentially increases while the cost of the storage rises by steps. Finally, the cost of reinforcement steadily increases but for a high share of VREs, it becomes the least-costly option. 


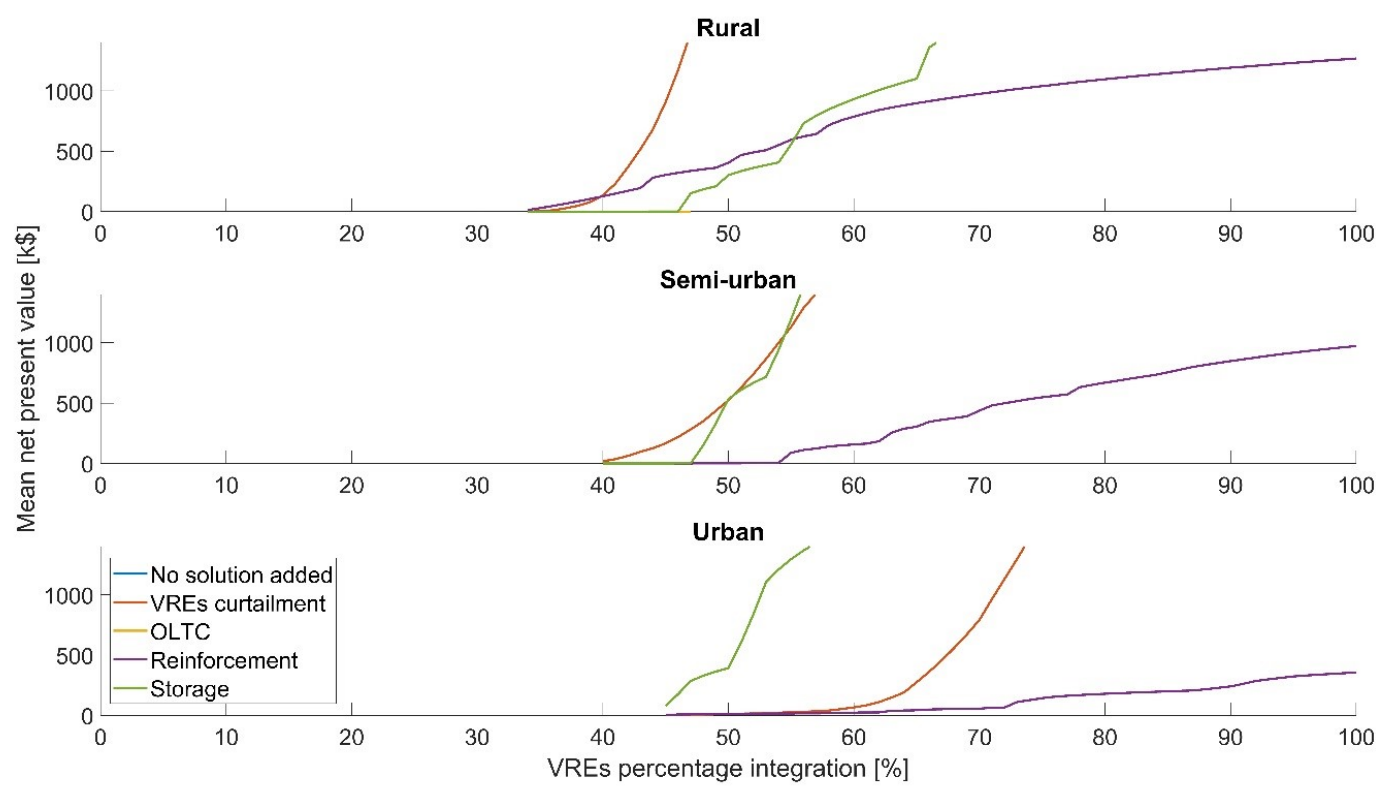

(a) Mean net present value of the modeled flexibility solutions $(\mathrm{k} \$$ ).

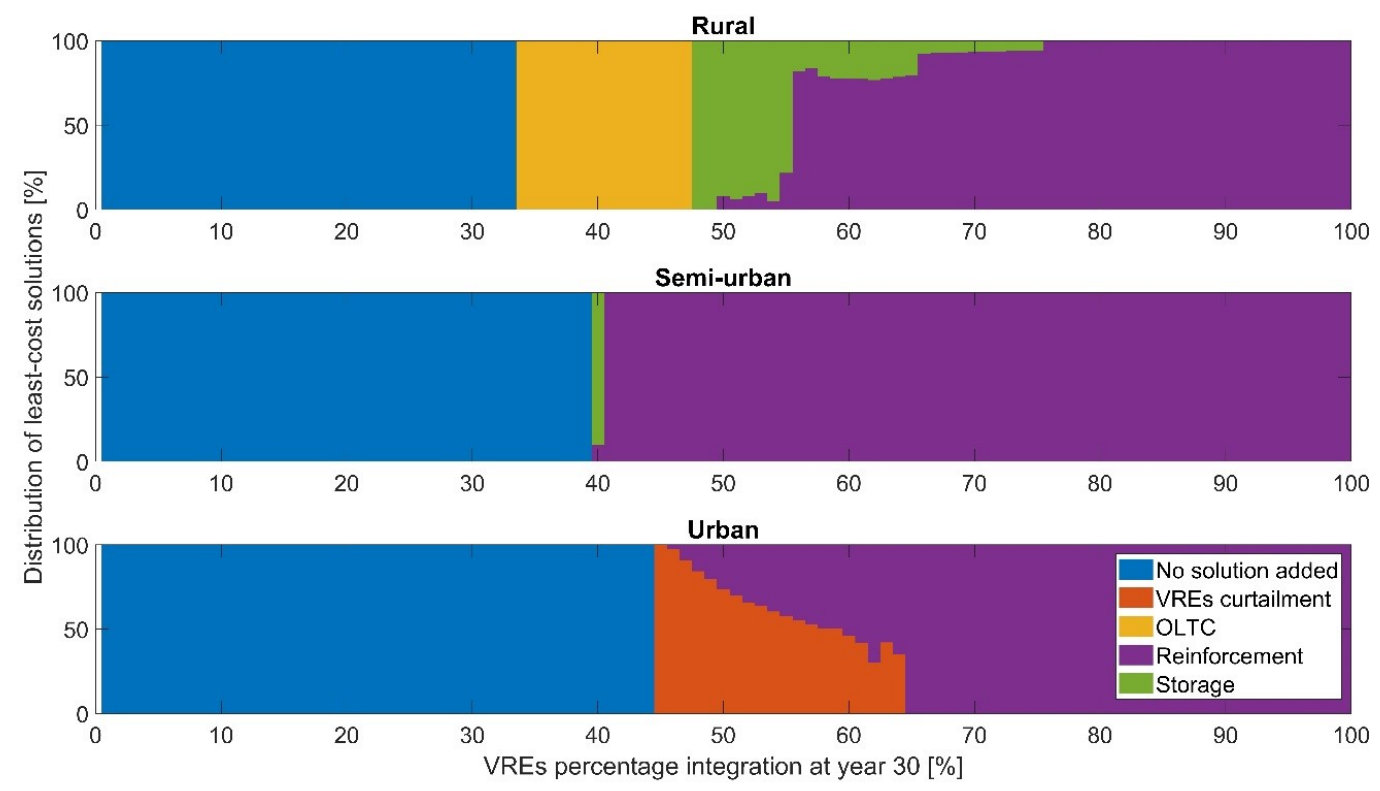

(b) Histogram of the least-costly flexibility solutions (\%).

Figure 11: Results of the Monte Carlo study on vREs integration on the three considered distribution grids. 
For the three distribution grids, the reinforcement is the most favored option if a high share of renewable is targeted. However, if the integration reaches $\sim 50 \%$, storage capacities is a good choice in rural areas while curtailing VRES production is preferable in urban areas. The histogram of the least cost solutions shown in Figure 11b confirms these conclusions. It can also be noted that, when there is no over-voltage or congestions issues, no flexibility solutions are needed.

As the rural distribution grid has some over-voltage issues, the oLTc is used until some congestions appear (it is the only distribution grid needed that flexibility lever). Then, storage solutions are the cheapest solution from $\sim 47 \%$ up to $\sim 55 \%$ of solar integration. However, from $\sim 55 \%$ to $\sim 65 \%$, reinforcing the grid is cheaper. This is the case when there is a fast integration of VRes at the beginning of the 30-years period. Therefore, the cost of installing the storage capacities plus the cost of replacement 15 years later exceed the cost of the reinforcement. With higher share of VRES, reinforcement is the only acceptable solution. For the semi-urban grid, storage capacities are preferred only at $\sim 40 \%$ of VRES penetration rate. Otherwise, reinforcing the grid is the least costly option. Finally, for the urban distribution grid, curtailing the vREs sources is the cheapest solution if the targeted share ranges from $\sim 45 \%$ up to $\sim 65 \%$. Meanwhile, reinforcement progressively becomes the cheapest solution as well for this category of distribution grid.

In the analyzed case study, the vRES sources only consisted of solar production, but wind turbines are also included in the medium-voltage grid. A sensitivity analysis has been performed with various energy mixes between solar and wind. The results show that, without any flexibility, the rural distribution grid can only integrate $\sim 30 \%$ of vRES while for the urban and semi-urban grids, it can reach up to $100 \%$. Concerning the flexibility levers, reinforcement is the preferred one for a high share of renewable and for the three categories of distribution grids. The storage capacities seem to be more interesting when the solar production is higher than the wind production. It can better store the surplus of energy and discharge during the off-peak periods for a less random production. Finally, curtailing production is a common solution in the urban grid but also in the rural one when wind production is high.

Grid investment mechanism. Based on the analysis of Figure 11, it can be observed that the represented distribution grids are well sized. The assumption of no reinforcement can be considered as relevant for the first 30-40 years of the simulation. Past that duration, no reinforcement is conducted on the distribution grids (only at the level of the transmission grid) because it would need a very long time to run the simulations (a matter of days). In addition, as it is very costly to reinforce these distribution grids, preliminary results have shown that the planning of reinforcements is done as far in time as possible which exceeds the time limit of the simulations (2000-2050).

\section{Integrating distribution grids in the European power system prospective model}

All the presented outcomes underline the fact that with the integration of vREs, the distribution grids face issues not considered by default in EUTGRID. In addition, because of the aggregation made in EUTGRID, considering only nodes at the transmission level, the presented flexibility solutions directly compete with more efficient technologies and the 
consequences are an under-estimation of the associated costs. Coupling EUTGRID with POLEs helps having better insights on the evolution of the transmission grid and its impacts on the European power system. To enhance the representation of more local flexibility levers, EUTGRID\&D includes representative distribution grids and is also coupled with POLES to increase the accuracy of the simulations.

\subsection{Considered scenarios}

To assess the role of the transmission grid, multiple scenarios with the same climate energy policy are set up [37]. They share the same socio-economic hypotheses, but they differ on the grid representation and the type of investments in the transmission grid [38]. From the scenarios considered in the coupling of poles and EutGRID. The one used in this work, called Clim-Dev, is a scenario where the infrastructure investments are made on an economic criterion and without any budget constraint. In this scenario, the grid is included, and a grid investment mechanism is being used. With the help of EUTGRID, grid requirements are determined based on congestions and the payback period for HVAC and HVAC cables. A $2{ }^{\circ} \mathrm{C}$ climate energy policy is also implemented, aiming at keeping the cumulative $\mathrm{CO}_{2 \text {-eq }}$ emissions under $1,300 \mathrm{GtCO}_{2 \text {-eq }}$ from 2011 up to 2100 as stated in [39].

A similar scenario, called Clim-Distrib, is run with EUTGRID\&D, coupled with Poles, with the same $2{ }^{\circ} \mathrm{C}$ climate energy policy. The results are compared region by region for their local emissions and the use of flexibility solutions as well as with the Clim-Dev scenario, where the distribution grids are aggregated per node (i.e. only the transmission grid is considered in the cost optimization) [40].

When considering just the distribution grids, vRes are arbitrarily increased with a fixed demand so that its share increases, controlling the power output. When considering the coupling of POLES and EUTGRID\&D, discussed in Section 5.4, the injected power is the result both from the investments decided in poLEs and the dispatch calculated in EUTGRID\&D.

\subsection{Restriction on the distribution grids representation}

To cover all the European demand, EUTGRID\&D needs almost 90,000 representative distribution grids. It would be possible to include them in EUTGRID\&D with representative distribution grids but the computational time would be unacceptable with today's computers capabilities. To illustrate this increase in time computation, EUTGRID\&D was run for one year with various representative distribution grids. For one year, less than 3 minutes are needed to simulate the representation of the European transmission grid. With just three nodes presenting a detailed distribution grids (at the three representative French nodes), the simulation takes almost 30 minutes (all other nodes of the transmission grid have aggregated consumption and production, i.e. the distribution grid is not considered). Each time three more distribution grids are added to the model the simulation is being increased by at least that duration (though it is not linear), which is simply not acceptable knowing that we aim at conducting prospective studies for the next 50 to 100 years considering a various set of scenarios over $\sim 100$ nodes for the equivalent European power system. 
For that reason, EUTGRID\&D only includes three representative distribution grids (rural, semi-urban and urban), presented in Section 4.1. The interaction with the transmission grid is thus considered for the connections between these three distribution grids and their neighbors. For all other nodes of the European transmission system, the distribution grids are aggregated, thus over-voltage or congestion are not modeled.

\subsection{At the level of the distribution grid}

The three distribution grids are associated to the region where they are connected. The Ile-de-France region with the representative urban distribution grid is referred to as the region IDF. The North region with the representative semiurban distribution grid is referred to as the region HDF (Hauts-de-France). The South region with the representative rural distribution is referred to as the region PACA (Provence-Alpes-Côtes d'Azur).

\subsection{1. $\mathrm{CO}_{2-e q}$ emissions per region}

For each region, the emissions are calculated in $\mathrm{MtCO}_{2-\mathrm{eq}}$. The results are displayed in Figure 12a for the region IDF, in Figure $12 \mathrm{~b}$ for the region HDF and in Figure 12c for the region PACA. It can be observed that the yearly emissions follow the same behavior in all regions, but they are greater in scenario Clim-Distrib. This rise can be explained with the increased detail of the distribution grids. New congestions and voltage level arise because of the vREs production and there is a stronger need for back-up capacities, which are more polluting.

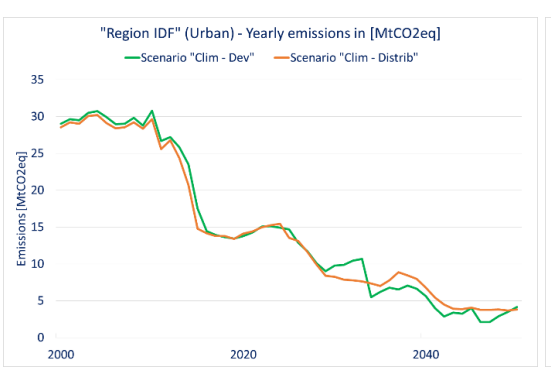

(a) Region IDF.

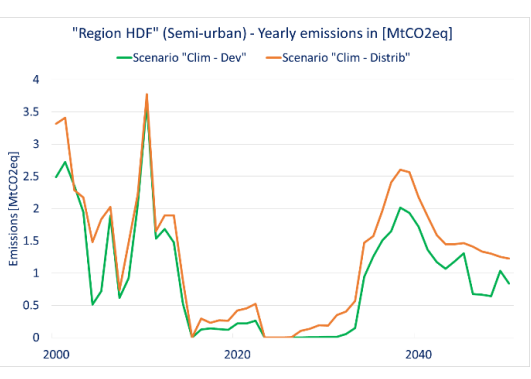

(b) Region HDF.

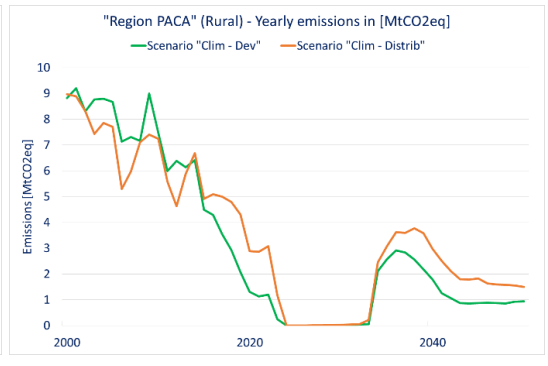

(c) Region PACA.

Figure 12: Yearly emissions for the two scenarios Clim-Dev and Clim-Distrib per region $\left(\mathrm{MtCO}_{2-\mathrm{eq}}\right)$.

In the HDF and PACA regions, it can be observed that the $\mathrm{CO}_{2-\mathrm{eq}}$ emissions decreases until 2020 to rise again and then stabilize in 2050. To understand these fluctuations, the energy mixes need to be assessed per region.

\subsubsection{Energy mix per region}

For the Clim-Dev and Clim-Distrib scenarios, the energy mix per region are displayed for the year 2010, 2030 and 2050 in Figure 13. The production is distinguished between nuclear, fossil with no ccs and with ccs, hydro, renewable (from geothermal and biomass) and vRes. Imports and exports are also displayed.

Various situations arise for the three regions. Region IDF, with the urban distribution grid, relies on importations to supply its increasing demand. The production from fossil fuel with no ccs is being replaced by more vREs production 


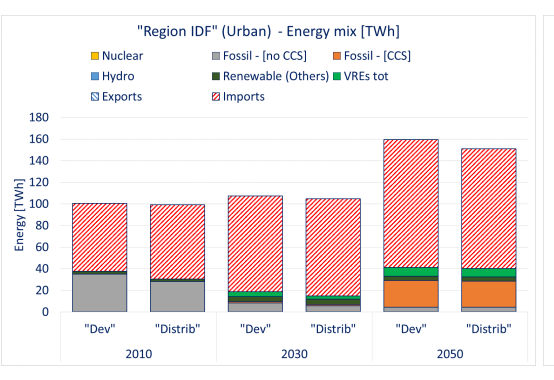

(a) Region IDF.

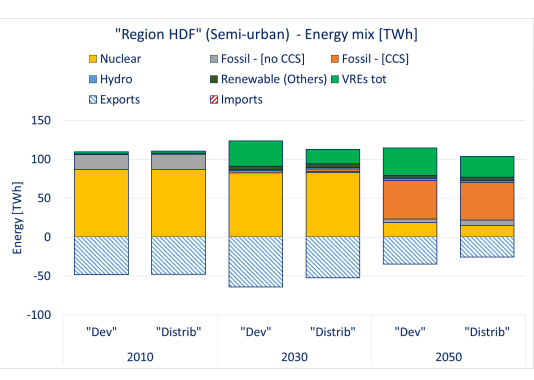

(b) Region HDF.

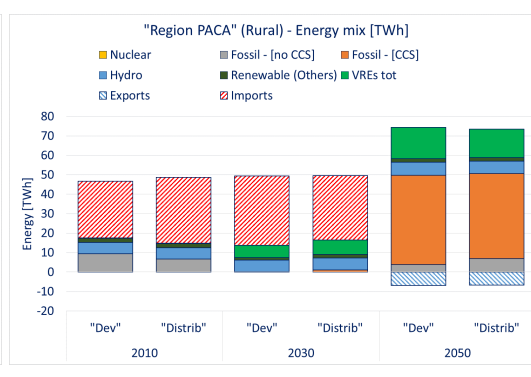

(c) Region PaCa.

Figure 13: Energy mix in 2010, 2030 and 2050 for the two scenarios Clim-Dev and Clim-Distrib per region (TWh).

and fossil technologies with ccs. In the region HDF, the nuclear production phases out after 2030 and pushes to introduce more VRES production and fossil technologies with cCS. In the region PACA, the same trend occurs, allowing the region to become a net exporter in 2100.

Between the two scenarios, some distinctions can be observed: there are less importations and lower exportations in Clim-Distrib than in Clim-Dev. Production from fossil fuel with no ccs also increases. These results indicate that the representation of the distribution grids modify the dispatching as more issues related to congestion and overvoltage need solving. It suggests also that a limited integration of vRES in the distribution grids reduces the exchanges between the neighboring regions.

\subsubsection{Flexibility solutions}

In the distribution grids, the flexibility solutions are the only ways of solving the congestion and over-voltage issues. The current analysis focuses on the storage technologies and the use of demand response. Curtailment of vRES production is also available but, in the scenario Clim-Distrib, there is no NDE. If compared with Clim-Dev, the region PACA has to curtail up to 6 TWh of vRES energy. To illustrate the differences between the two scenarios, Figure 14 shows the production from storage technologies from 2000 to 2050 and Figure 15 displays the demand shifted via the demand response technologies during the same period. For both the storage and demand response, the produced energy is higher for the region IDF which is more populated and in similar levels for the two other regions.

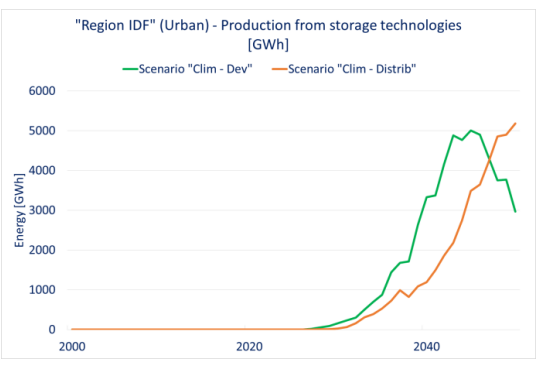

(a) Region IDF.

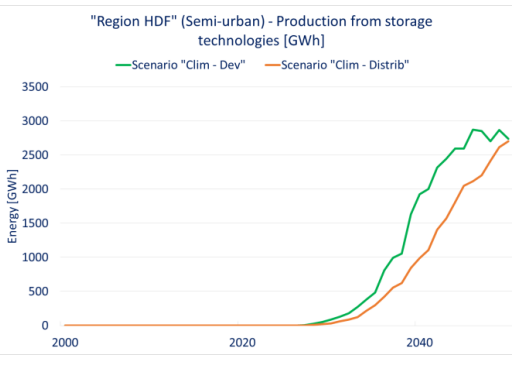

(b) Region HDF.

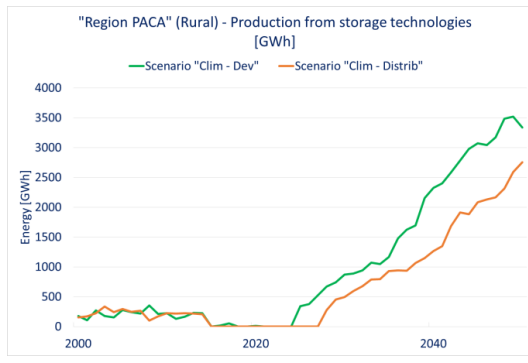

(c) Region PACA.

Figure 14: Production from storage technologies in 2010, 2030 and 2050 for the two scenarios Clim-Dev and Clim-Distrib per region (GWh). 
Concerning the production from storage technologies, it can be observed that it increases similarly in all regions and for the two scenarios. However, there is a delay between Clim-Dev and Clim-Distrib caused by the allocation of the stationary batteries and $\mathrm{v}_{\mathrm{G}} \mathrm{G}$ technologies in the distribution grid. A small part of the regional capacities is distributed among the nodes in the distribution grid. During the dispatch, the use of these storage capacities is limited by the congestions and the over-voltage issues in the distribution grid. Therefore, their usage is being reduced in the scenario Clim-Distrib compared to the scenario Clim-Dev.

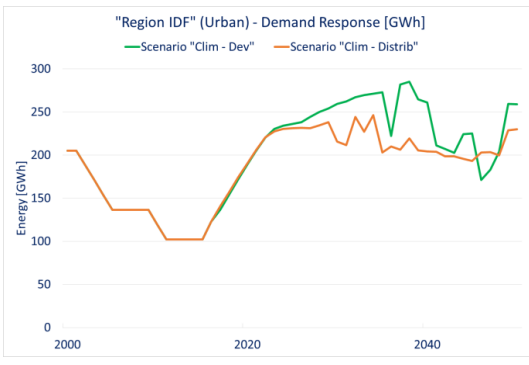

(a) Region IDF.

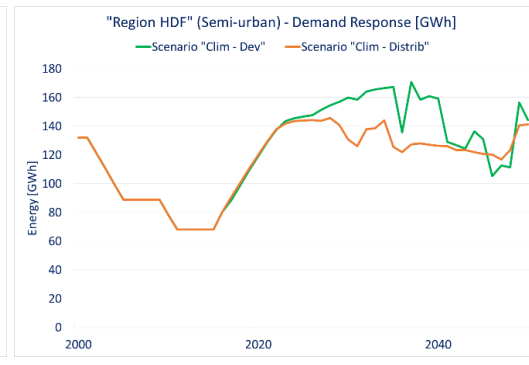

(b) Region HDF.

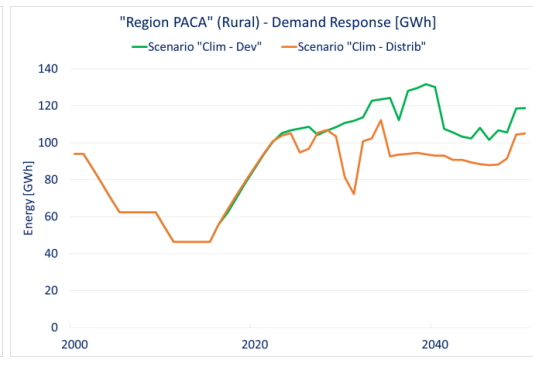

(c) Region PACA.

Figure 15: Load shifting as demand response in 2010, 2030 and 2050 for the two scenarios Clim-Dev and Clim-Distrib (GWh).

A similar result can be observed when the yearly load shifting is being analyzed for the two scenarios and for the three regions. In the Figure 15, the amount of energy shifted is also lower. As the demand response potential is only limited to $5 \%$ of the peak demand, its usage is still needed, and the difference becomes less visible than with storage technologies.

\subsection{European transmission grid infrastructure}

All the modifications observed in the $\mathrm{CO}_{2-\mathrm{eq}}$ emissions, the energy mixes and the flexibility options reduce exchanges between regions. Consequently, the transmission grid requirements are modified. The tool coupling POLES and EUTGRID\&D provides, per region (i.e. for each node), a summary of the investments planned for a given period (in our case, from 2000 to 2050). The investments depend on the evolution of the nodal consumption and production, as well as technologies, losses, flexibility levers, etc. In this context, focusing on regions where a distribution grid has been represented, and comparing it with the same case without distribution grid, highlights the impact on the evolution of the power system when aggregating or not the distribution grid per node.

The development of the grid infrastructure is quantified in TWkm. It multiplies the capacity reinforcement by the length of the lines. This indicator helps comparing situations: for example, between a small line that is upgraded numerous times and a long line that is reinforced only once.

\subsubsection{Interconnections of the analyzed regions}

For the regions IDF and HDF, the investments planned during 2010-2030 by ENTSO-E are enough for the period 20302050. But for the region PACA, the reinforcements it is different. Figure 16 shows the number of interconnections for 
the region PACA from 2000 up to 2050 for the two scenarios Clim-Dev and Clim-Distrib.

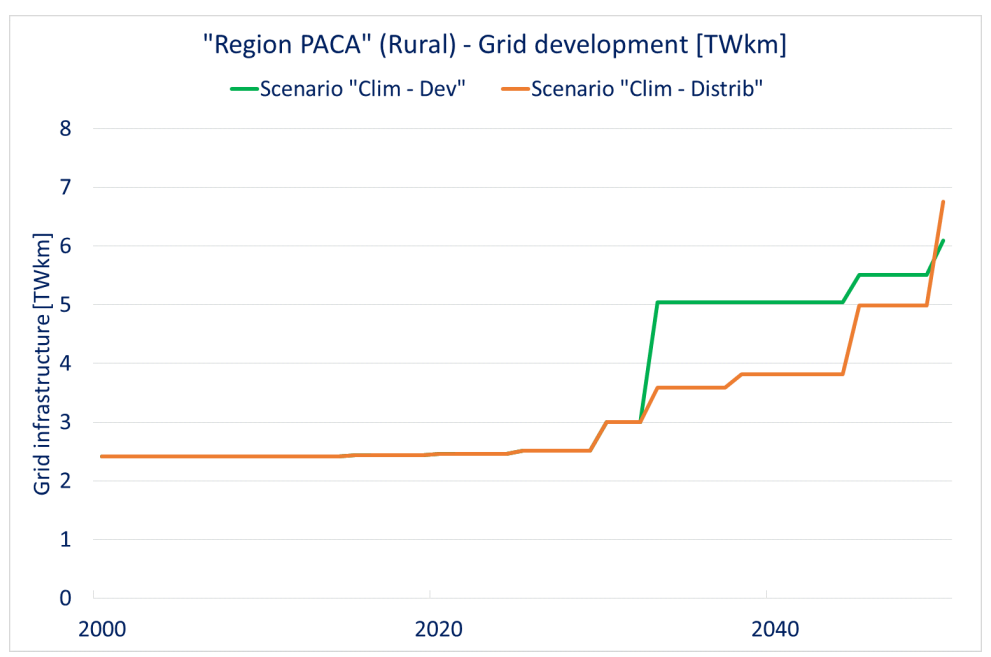

Figure 16: Transmission grid interconnections for the PACA region from 2000 up to 2050 for the two scenarios Clim-Dev and Clim-Distrib (TWkm).

In the end, the grid interconnections reach the same level with almost $6 \mathrm{TWkm}$. However, the timing of the reinforcement is different: in 2033 for Clim-Dev and in 2043 and 2048 for Clim-Distrib. The difference is due to the fact that 6 TWh of VREs production are still spilled in Clim-Dev and a reinforcement is useful to integrate this cheap energy. Moreover, HVDC technologies is chosen to integrate this large amount of energy. In scenario Clim-Distrib, the reinforcement decisions follow the load increase and only with HVAC technologies. The representation of the three distribution grids suggests that it can have a significant impact on the evolution of the transmission grid.

\subsubsection{Transmission grid development at the European level}

The development of the transmission grid is determined at the European level and compared to the scenario Clim-Dev. The findings confirm the important changes both in terms of total grid requirements and technologies chosen between HVAC and HVDC. However, it should be recalled that the modeling framework is limited to only three representative distribution grids, which have been included in the European sector (on three nodes in France) and nowhere else in the modeled European power system. We can only imagine the changes that could come out of a full integration of distribution grids in each nodes of the European transmission grid.

Figure 17 shows the developments of the European transmission grid for the two scenarios Clim-Dev and ClimDistrib. Up to 2030, the investments are identical. Then the development of the European transmission grid is lower in scenario Clim-Distrib. It reaches 563 TWkm in 2050 while in Clim-Dev the grid has been upgraded up to 594 TWkm.

To further analyze the impact of representing the distribution grids for three nodes of the representation of the European transmission grid, the requirements of the two scenarios can be compared for the period 2030-2050. The results are gathered in Table 2. The reinforcement requirements are lower for the Clim-Distrib scenario. It can also be observed a clear shift between the two scenarios regarding the infrastructure. The Clim-Dev scenario leads to a 


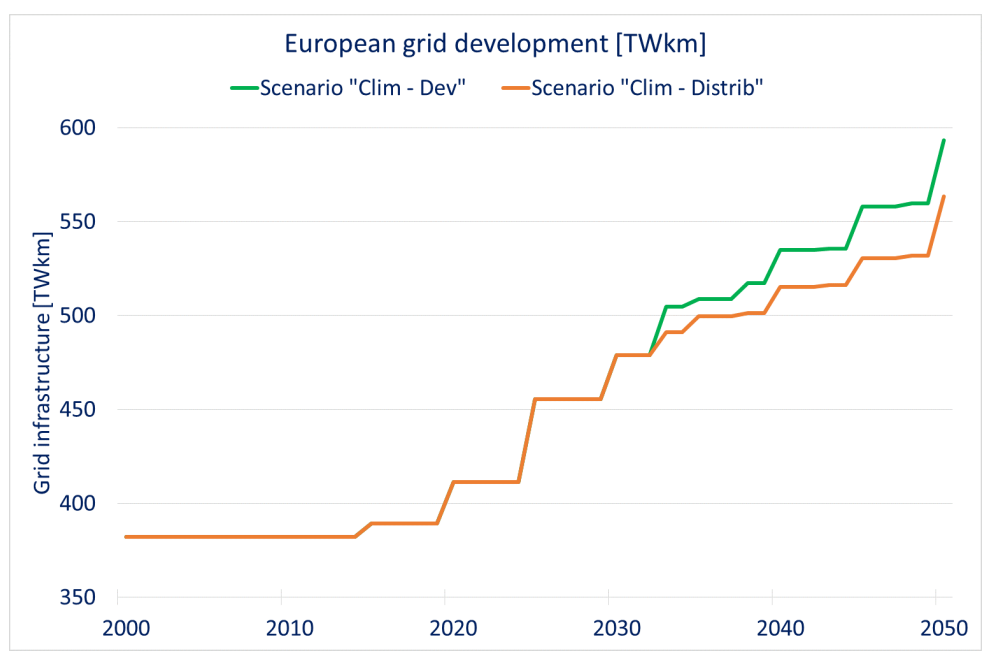

Figure 17: European transmission grid infrastructure from 2000 up to 2050 for the two scenarios Clim-Dev and Clim-Distrib (TWkm).

grid mostly upgraded with HVDC technologies. On the opposite, the Clim-Dev scenario leads to reinforcements with HVAC. However, this distribution is closer to the distribution from 2010-2030. Finally, the total budget needed for these reinforcements is lower for the Clim-Distrib scenario, with $\$ 7.2$ billion/year compared to $\$ 9.7$ billion/year for the Clim-Dev scenario.

Table 2: Transmission grid investments and infrastructures for the two scenarios Clim-Dev and Clim-Distrib.

\begin{tabular}{lccc}
\hline & $2010-2030$ & Clim-Dev & 2030-2050 \\
\hline Grid added (TWkm) & 97 & 114 & Clim-Distrib \\
- HVAC (\%) & 74 & 30 & 85 \\
- HVDC (\%) & 26 & 70 & 69 \\
Investment (billion $\$)$ & 180 & 194 & 31 \\
& $(\sim 9$ billion/year $)$ & $(\sim 99.7$ billion/year $)$ & $(\sim \% 7.2$ billion/year $)$ \\
\hline
\end{tabular}

These outputs are the consequences of a cascade effect due to the integration of the three distribution grids. The power flows around the regions IDF, HDF and PACA are modified and consequently, it impacts the neighboring regions and then, on the long term, the overall Europe.

The impact of considering three distribution grids, through EUTGRID\&D, can also be observed on the location of the reinforcement projects. Figure 18 displays two maps of the transmission grid reinforcements for the period 20302050, illustrating the scenario Clim-Dev and Clim-Distrib, with both HVAC and HVDC lines creation.

The location of the reinforcements differs between the two scenarios. In Clim-Dev, Europe is being more interconnected with a focus on the connection in the North Sea. However, in Clim-Distrib, there is a concentration 


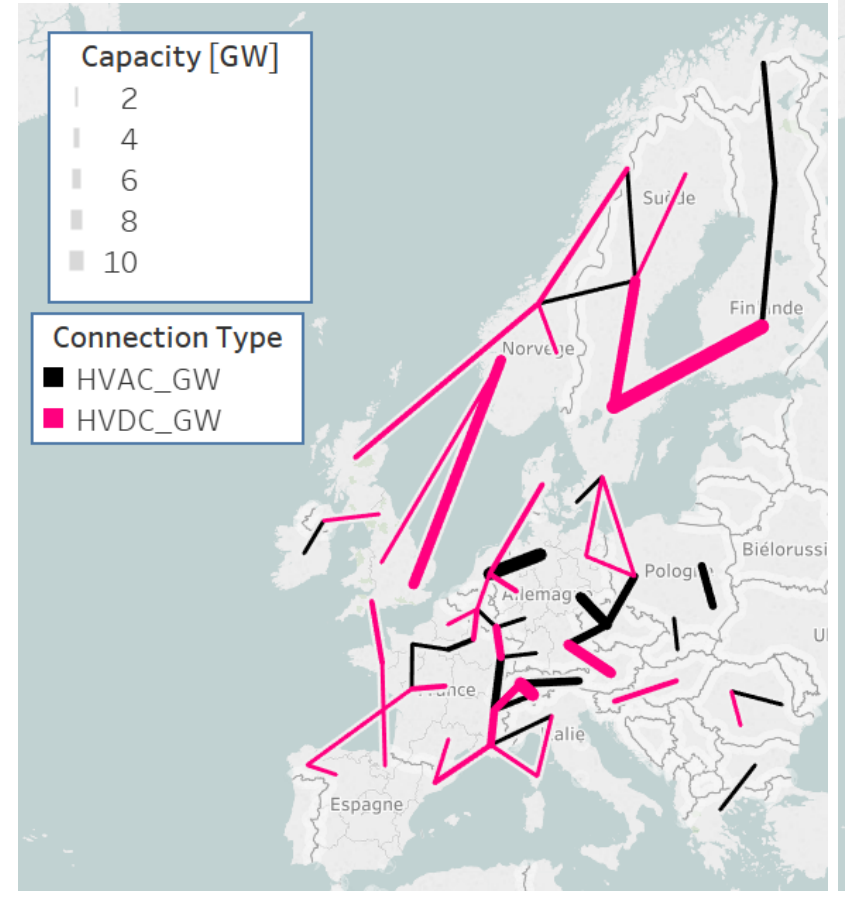

(a) Scenario Clim-Dev.

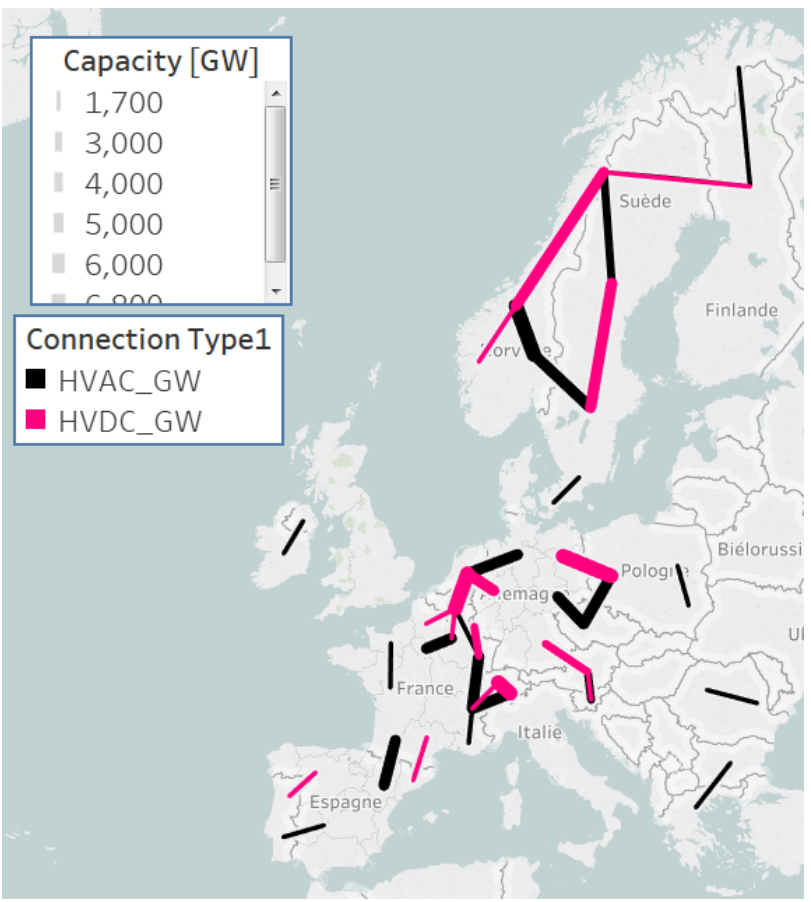

(b) Scenario Clim-Distrib.

Figure 18: Transmission grid lines creation for the period 2030-2050 when considering distribution grids in three nodes in France (GW).

of reinforcement project in three areas. The main corridor connects Benelux, France, Germany and Switzerland. Then the Scandinavian area is being highly reinforced. Finally, interconnections between Germany and Poland are upgraded to solve the congestions provoked by the German off-shore wind production. The French-Spanish interconnection is an important corridor to integrate the Spanish solar production. Despite their differences, these two scenarios have some reinforcement projects in common such as the Benelux corridor, the Scandinavian area and the French-Spanish interconnection. These findings underline the fact that it exists various options for the development of the European grid infrastructure, which are clearly sensitive to the level of accuracy in the representation of the physical infrastructure, in addition to the economic outlook models.

\section{Conclusions}

As most of the VRES installed capacities are connected to the distribution grids, new issues arise which complicate the operation of the power system, for instance congestion and over-voltage. As grids cover all Europe, the large integration of VRES affects the operation of the transmission and distribution grids. For this reason, the European Transmission Grid Investment and Dispatch (EUTGRID) model has been improved with the implementation of linearized AC equations to represent voltage and reactive power.

The new model, called European Transmission Grid and Dispatch with Distribution grid representation (EUTGRID\&D) is coupled with the Prospective Outlook on Long-term Energy Systems (POLES) model, to analyze the issues in three 
representative distribution grids as the share of VRES increases. In rural grids, over-voltage situations occur first and then congestions. In semi-urban and urban grids, only congestions appear. To solve these issues, flexibility solutions are used, and their net present values are compared for targeted shares of vREs. The results show that, for high share of VRES (above $60 \%$ ) reinforcement is the preferred solution for the three considered distribution grids. For lower VRES penetration rates, the curtailment of VRES production or installation of storage capacities can be cheaper. A combination of the presented flexibility solutions might be more realistic, but the results indicate already the interest of including the distribution grids models in the European power system prospective study when facing issues with the large-scale integration of vREs.

To understand how the inclusion of representative distribution grids affects the outputs of the long-term energy outlook of the European power system, a scenario based on the European $2{ }^{\circ} \mathrm{C}$ climate energy policy, coupling EuTGRID\&D with POLES, is assessed. The emissions within the regions increase as more back-up technologies are needed in that case. It is confirmed with the analysis of the energy mixes, showing a reduction of energy import and export and an increase in production from fossils with no ccs. The use of flexibility options increases but with a delay: the batteries located in the distribution nodes are limited by the congestions and cannot be used for flexibility at a larger scale.

The consequence of the modification of the energy dispatch within each region is a slower development of the transmission grid. It affects also the choices of reinforcement with a shift from HVDC to HVAC around the regions including the representative distribution grids. At the European level, a cascade effect can be observed together with an amplification of these findings: the neighboring regions see a modification of the exchanges and consequently modify their energy dispatch.

The next logical step is to increase the number of nodes where the distribution grid is represented and not aggregated. This requires either a significant increase in computational power, or a complete change in the way the problem is solved during the optimizations. This also opens a second challenge: how to correctly determine the characteristic of the distribution grid that is integrated per node. There are multiple possibilities here: a typical grid or a simplification of the real one (which is a tremendous work as data needs to be gathered for many nodes in various countries). It is anyway a very motivating challenge to be addressed!

\section{Acknowledgments}

This work was funded by the ARC-4 Energies Région Auvergne Rhône-Alpes from France.

\section{References}

[1] T. Ackermann, V. Knyazkin, Interaction between distributed generation and the distribution network: operation aspects, in: IEEE/PES Transmission and Distribution Conference and Exhibition, Vol. 2, 2002, pp. 1357-1362.

[2] SWECO, Study on the effective integration of distributed energy resources for providing flexibility to the electricity system, techreport 54697590000, Final report to The European Commission (Apr. 2015). 
URL https://ec.europa.eu/energy/sites/ener/files/documents/5469759000EffectiveintegrationofDERFinalver2_ 6Apri12015.pdf

[3] C. L. Masters, Voltage rise: the big issue when connecting embedded generation to long 11 kv overhead lines, Power Engineering Journal 16 (1) (2002) 5-12. doi:10.1049/pe:20020101.

[4] L. M. Cipcigan, P. C. Taylor, Investigation of the reverse power flow requirements of high penetrations of small-scale embedded generation, IET Renewable Power Generation 1 (3) (2007) 160-166.

[5] M. Liserre, T. Sauter, J. Y. Hung, Future energy systems: integrating renewable energy sources into the smart power grid through industrial electronics, IEEE Industrial Electronics Magazine 4 (1) (2010) 18-37.

[6] S. Sayeef, S. Heslop, D. Cornforth, T. Moore, S. Percy, J. Ward, A. Berry, D. Rowe, Solar intermittency: Australia's clean energy challenge. Characterising the effect of high penetration solar intermittency on Australian electricity networks, techreport, Commonwealth Scientific and Industrial Research Organisation (CSIRO) (Jun. 2012).

URL https://publications.csiro.au/rpr/download?pid=csiro:EP121914\&dsid=DS1

[7] K. Fekete, Z. Klaic, L. Majdandzic, Expansion of the residential photovoltaic systems and its harmonic impact on the distribution grid, Renewable Energy 43 (2012) 140-148.

[8] L. Collins, J. K. Ward, Real and reactive power control of distributed PV inverters for overvoltage prevention and increased renewable generation hosting capacity, Renewable Energy 81 (2015) 464-471. doi : 10.1016/j.renene.2015.03.012.

[9] E. Reihani, M. Motalleb, R. Ghorbani, L. S. Saoud, Load peak shaving and power smoothing of a distribution grid with high renewable energy penetration, Renewable Energy 86 (2016) 1372-1379. doi:10.1016/j.renene.2015.09.050.

[10] R. A. Verzijlbergh, L. J. D. Vries, Z. Lukszo, Renewable energy sources and responsive demand. Do we need congestion management in the distribution grid?, IEEE Transactions on Power Systems 29 (5) (2014) 2119-2128.

[11] C. Lueken, P. M. S. Carvalho, J. Apt, Distribution grid reconfiguration reduces power losses and helps integrate renewables, Energy Policy 48 (2012) 260-273.

[12] A. Schroeder, Modeling storage and demand management in power distribution grids, Applied Energy 88 (12) (2011) $4700-4712$.

[13] J. Després, N. Hadjsaid, P. Criqui, I. Noirot, Modelling the impacts of variable renewable sources on the power sector: Reconsidering the typology of energy modelling tools, Energy 80 (2015) 486-495.

[14] N. E. Koltsaklis, A. S. Dagoumas, Incorporating unit commitment aspects to the European electricity markets algorithm: An optimization model for the joint clearing of energy and reserve markets, Applied Energy 231 (2018) 235-258. doi:https://doi.org/10.1016/j. apenergy.2018.09.098.

[15] N. E. Koltsaklis, A. S. Dagoumas, State-of-the-art generation expansion planning: A review, Applied Energy 230 (2018) 563-589. doi: $10.1016 / j$.apenergy. 2018.08.087

[16] N. E. Koltsaklis, A. S. Dagoumas, M. C. Georgiadis, G. Papaioannou, C. Dikaiakos, A mid-term, market-based power systems planning model, Applied Energy 179 (2016) 17-35. doi:https://doi.org/10.1016/j.apenergy.2016.06.070.

[17] R. Loulou, U. Remne, A. Kanudia, A. Lehtila, G. Goldstein, Documentation for the TIMES model, Part I, techreport, Times (2005).

[18] E3MLab, The PRIMES model, techreport, E3MLab (2017).

[19] A. Fuchs, T. Demiray, E. Panos, R. Kannan, T. Kober, C. Bauer, W. Schenler, P. Burgherr, S. Hirschberg, ISCHESS - Integration of stochastic renewables in the Swiss electricity supply system, Tech. rep., ETH Zurich - Research Center for Energy Networks. PSI - Laboratory for energy systems analysis (2017).

[20] P. Criqui, S. Mima, P. Menanteau, A. Kitous, Mitigation strategies and energy technology learning: An assessment with the POLES model, Technological Forecasting and Social Change 90 (2015) 119-136.

[21] S. Allard, S. Mima, V. Debusschere, T.-T. Quoc, P. Criqui, N. Hadjsaid, Large scale integration of variable renewable energies in the European power system: a model POLES-EUTGRID based approach, in: International Energy Workshop (IEW), 2018, pp. 1-6.

[22] A. N. Espinosa, Low voltage networks models and low carbon technology profiles, Ph.D. thesis, The University of Manchester (Jun. 2015).

[23] Distribution System Operators Observatory, JRC smart electricity systems and interoperability, Online, accessed: 24-March-2020 (2020). 
URL https://ses.jrc.ec.europa.eu/distribution-system-operators-observatory

[24] G. Prettico, F. Gangale, A. Mengolini, A. Lucas, G. Fulli, Distribution system operators observatory, techreport, European Commission Joint Research Centre (2016).

[25] S. Allard, S. Mima, V. Debusschere, Q. T. Tran, P. Criqui, N. Hadjsaid, European transmission grid expansion as a flexibility option in a scenario of large scale variable renewable energies integration, Energy Economics (2020). doi:https://doi.org/10.1016/j.eneco. 2020.104733.

[26] A. S. Dagoumas, M. L. Polemis, An integrated model for assessing electricity retailer's profitability with demand response, Applied Energy 198 (2017) 49-64. doi:https : //doi.org/10.1016/j. apenergy . 2017.04.050.

[27] F. Hinz, Voltage stability and reactive power provision in a decentralizing energy system: a techno-economic analysis, Ph.D. thesis, Technische Universität Dresden, Fakultüt der Wirtschaftswissenschaften, Lehrstuhl für Energiewirtschaft (Dec. 2017).

[28] R. D. Zimmerman, C. E. Murillo-Sanchez, R. J. Thomas, MATPOWER: Steady-state operations, planning, and analysis tools for power systems research and education, IEEE Transactions on Power Systems 26 (1) (2011) 12-19.

[29] M. Cui-Mei, G. Quan-Sheng, Method for calculating CO2 emissions from the power sector at the provincial level in china, Advances in Climate Change Research 5 (2) (2014) 92-99.

[30] P. Criqui, S. Mima, European climate-energy security nexus: A model based scenario analysis, Energy Policy 41 (2012) 827-842.

[31] Enedis, Description physique du réseau public de distribution, techreport, NOI-RES-07E (Mar. 2017).

[32] SMA, Maximum freedom when oversizing - More flexibility and higher profitability for PV projects with sunny central inverters, Tech. rep., White paper (May 2018).

[33] R. L. Fares, M. E. Webber, What are the trade-offs between battery energy storage cycle life and calendar life in the energy arbitrage application?, Energy Storage 16 (2018) 37-45.

[34] B. P. Swaminathan, Operational planning of active distribution networks-Convex relaxation under uncertainty, Ph.D. thesis, Université Grenoble Alpes (2017).

[35] E-CUBE Strategy Consultants, Etude sur la valeur des flexibilités pour les réseaux de distribution, Tech. rep., Commission de Régulation de l'énergie (Jan. 2016).

[36] Commission de régulation de l'énergie, Open Data, Online, accessed: 24-March-2020 (2020). URL https: //www.cre.fr/Pages-annexes/open-data

[37] J.-N. Louis, S. Allard, V. Debusschere, S. Mima, T. Tran-Quoc, N. Hadjsaid, Environmental impact indicators for the electricity mix and network development planning towards 2050 - A POLES and EUTGRID model, Energy 163 (2018) 618-628. doi : 10.1016/j .energy . 2018.08 .093

[38] J.-N. Louis, S. Allard, F. Kotrotsou, V. Debusschere, A multi-objective approach to the prospective development of the European power system by 2050, Energy (2019). doi:10.1016/j .energy .2019.116539.

URL http://www.sciencedirect.com/science/article/pii/S0360544219322340

[39] L. Meyer, S. Brinkman, Climate Change 2014: Synthesis report. Contribution of working groups I, II and III to the fifth assessment report of the intergovernmental panel on climate change, resreport, IPCC, Synthesis Report (2014).

[40] S. Allard, S. Mima, V. Debusschere, T.-T. Quoc, P. Criqui, N. Hadjsaid, Long-term transmission capacity planning in a scenario with high share of variable renewable energies, in: AIEE Symposium on Energy Security - Current and Future Challenges to Energy Security, Vol. 1, International Association for Energy Economics, Rome, Italy, 2017, pp. 145-147. 